\title{
The Coulomb Resonances, the Quasi-Real Photons and Electro-Disintegration of Nuclei by High-Energy Electrons
}

\author{
A. A. Pasichnyi ${ }^{1}$, O. A. Prygodiuk ${ }^{2}$ \\ ${ }^{1}$ Institute for Nuclear Research, Kyiv, Ukraine \\ ${ }^{2}$ Taras Shevchenko Kyiv National University, Kyiv, Ukraine \\ Email: apasichny@kinr.kiev.ua
}

Received October 3, 2012; revised November 5, 2012; accepted November 15, 2012

Copyright (C) 2013 A. A. Pasichnyi, O. A. Prygodiuk. This is an open access article distributed under the Creative Commons Attribution License, which permits unrestricted use, distribution, and reproduction in any medium, provided the original work is properly cited.

\begin{abstract}
Various aspects of the influence of the quasi-real photons and the Coulomb resonances on the formation of the crosssection of inelastic scattering of high energy electrons on atomic nuclei are investigated. $E_{m i s s}$ is the energy that disappears in the processes of knocking-on of protons in the reactions $A\left(e, e^{\prime} p\right)(A-1)$. A new hypothesis that interprets the origin of the energy losses is proposed. Specific experiments that can confirm or refute this hypothesis are proposed as well. The "regularized" cross-sections of electro-disintegration of nuclei by high-energy electrons $\left(\sigma^{r e g}\left(\omega, \theta^{\prime}\right)\right)$ are calculated in the framework of the nuclear shell model. It is shown that for the experimental verification of the existence of Coulomb resonances, it is necessary to investigate the $\left(e, e^{\prime}\right)$ processes at relatively small angles of scattering. The peculiarities of numerical methods that are crucial in the investigation of inelastic scattering of high-energy electrons on nuclei in the framework of the nuclear shell model are analyzed in this work as well. The cross-sections of the scattering of high-energy electrons on the angle $\theta^{\prime}=0$ are calculated. It is shown that the orthogonality of the wave functions of a knocked-on proton in the initial and final states plays an important role in the interpretation of this process.
\end{abstract}

Keywords: Coulomb Resonance; Quasi-Real Photon; Inelastic Scattering; High-Energy Electron; Electro-Disintegration; Nuclear Shell Model; Numerical Method; Inelastic Scattering; Quasi-Elastic Peak

\section{Introduction: The Electro-Disintegration of Atomic Nuclei by High-Energy Electrons}

Due to the relative weakness of the electromagnetic and weak interactions of electrons, positrons, muons and neutrinos with nuclei, the structure investigation of atomic nuclei in the processes of elastic and inelastic scattering of these particles on nuclei [1-27] provides the most reliable information on various aspects of the structure of atomic nuclei. The above statement is based on the relatively high reliability of the information about the properties of electromagnetic and weak interactions, as well as the relatively high accuracy of the perturbation theory, in the framework of which (impulse approximation) we interpret the inelastic scattering of high-energy leptons by nuclei. The technical perfection reached at the moment in forming the monoenergetic high-energy elec- trons beams, as well as in registering these particles in nuclear experiment, played a decisive role in the choice of particles-projectiles as means of external influence on the atomic nucleus for studying the structure and properties of nuclei: they were certainly the high-energy electrons.

It is important to note that the structure of nuclei is investigated in this paper in the framework of the nuclear shell model (LS-coupling, independent particles). In the framework of this nuclear shell model, we will study and interpret the features of such unusual phenomena as the Coulomb resonances and the quasi-real photons in the aspect of their influence on the dynamics of electro-disintegration of nuclei. In other words, the aim of this paper is the investigation of possibilities of visualization and identification of Coulomb resonances in experimental studies of nuclear electro-disintegration. 
It must be emphasized that the Coulomb resonances are the something more than merely the Coulomb resonances. First, the Coulomb resonances present a practically essential part of the quantum-mechanical theory of quasi-discrete spectrum in non-relativistic quantum mechanics [12,25,27]. Second, the Coulomb resonances are, first and foremost, a natural extension of the nuclear shell model to the continuous spectrum region [12,25,27]. Third, it is the Coulomb resonances that will provide additional and very useful insight into our understanding of gigantic dipole resonance phenomenon in the framework of the shell model [12,25,27], etc. Finally, the reader may remember $[12,25,27]$ that at this stage the Coulomb resonances and the quasi-real photons are investigated exclusively poorly. However, in both theory of inelastic highenergy electron scattering and theory of atomic nuclei, the Coulomb resonances and the quasi-real photons are phenomena that can manifest itself in many phenomena and experiments of nuclear physics [12,25,27].

We suppose that the study of the reactions of proton knockout $A\left(e, e^{\prime} p\right)(A-1)$ and neutron knockout $A\left(e, e^{\prime} n\right)(A-1)$ from various atomic nuclei presents a particular interest just in the framework of the nuclear shell model. From this point on, we shall assume that the process of inelastic scattering of a high-energy electron at a nucleus is accompanied by transfer of energy $\omega=\varepsilon-\varepsilon^{\prime}$ $\left(\omega>0 ; \varepsilon\right.$ and $\varepsilon^{\prime}$ are the initial and final energies of the scattered electron), and momentum $\boldsymbol{q}=\boldsymbol{k}-\boldsymbol{k}^{\prime}$ to the nucleus. $\boldsymbol{k}$ and $\boldsymbol{k}^{\prime}$ are the electron momenta before and after the act of inelastic collision of the electron and the nucleus. We also suppose that the process of inelastic collision of the electron with the nucleus in the investigated region of the kinematic variables $\omega, \boldsymbol{q}$ is caused mainly by a collision of the scattered electron with a single nucleon of the nucleus.

As a result of this collision, one of the nucleons of the atomic nucleus, having received the required energy $\omega$ in this act, overcomes the action of attractive nuclear forces and flies out from the atomic nucleus A with momentum $\boldsymbol{K} \equiv \boldsymbol{K}_{x} \quad(x=p$ and $x=n$ in the cases of knocking out of a proton and a neutron, respectively) and energy $E \equiv E_{x}:\left|\boldsymbol{K}_{x}\right|=\sqrt{2 M E_{x}}$.

Note that according to the conservation laws of momentum and energy, the exact expression for the distribution of the transferred electron energy $\omega$ between the nucleus $A-1$ and the knocked-out nucleon has the following form (here and in the following $\hbar=c=1$ [28]):

$$
\omega=w_{x v l}+\frac{\boldsymbol{q}^{2}}{2 A M}+\frac{\left(\boldsymbol{K}-\frac{\boldsymbol{q}}{A}\right)^{2}}{2 \bar{M}_{p}} \equiv w_{x v l}+T_{A}+T_{(p, A-1)} .
$$

The new quantities appearing in Equation (1) are as follows: $w_{x v l}$ is the energy required for ejection of nucleons from the $x v l$-shell of an infinitely heavy nucleus (the separation energy of the nuclear $x v l$-nucleon); $T_{A}$ is the energy of motion of the center of mass of the target nucleus $A$ after its collision with the scattering electron; $T_{(p, A-1)}$ is the energy of relative motion of the knockedout proton and the residual nucleus $A-1 ; \bar{M}_{p}=\frac{A-1}{A} M$ is the reduced mass of the proton.

We point out that Equation (1) could be written in the following form as well:

$$
\omega=\frac{\boldsymbol{K}^{2}}{2 \bar{M}_{p}}+w_{x v l}^{e f f},
$$

where the effective separation energy $w_{x v l}^{e f f}$ is defined by the following apparent formula:

$$
w_{x v l}^{e f f}=w_{x v l}+\left[\frac{\mathbf{q}^{2}}{2 A M}+\frac{\mathbf{q}^{2}}{2 A^{2} \bar{M}_{p}}-\frac{\mathbf{K} \cdot \mathbf{q}}{A \bar{M}_{p}}\right]
$$

It is important to point out that, according to Equation (3), the effective separation energy $w_{x v l}^{e f f}$ of the $x v l$ nucleon depends somewhat on the kinematics of experiment. In the limit case of $A \gg 1$ we have:

$$
T_{(p, A-1)}=E_{p}=\frac{\boldsymbol{K}^{2}}{2 M} ; \quad w_{x v l}^{e f f}=w_{x v l} .
$$

The effective separation energy $w_{x v l}^{e f f}$ in the approximation $\left(A \gg 1, \bar{M}_{p}=M\right)$ will be identified in the future numerical calculations with the binding energy of the nucleon $\varepsilon_{x v l}$ in the nuclear shell. There is a good reason to believe that the calculations of the cross sections of nuclear electro-disintegration of heavy, medium and even light nuclei, which are performed in this approximation, will be quite acceptable for preliminary conclusions.

A nucleon knocked out from a nucleus moves in the average field $V_{A x}(r)$ of this nucleus in both the bound state and the state of continuous spectrum. In the shell model the knocked-out nucleon dynamics in the bound state is presented by the wave functions $\psi(\boldsymbol{r})=\varphi_{x v l m}(\boldsymbol{r})$ calculated in average field $V_{A x}(r)$; here $v=1,2,3, \cdots$ is the radial quantum number; $l=0(s), 1(p), 2(d), 3(f), \cdots$ is the orbital quantum number. It is reasonable to calculate the continuous spectrum wave functions

$\psi(\boldsymbol{r})=\psi_{\boldsymbol{K}}^{ \pm}(\boldsymbol{r})$ in the same average field $V_{A x}(r)$ In this case the bound state wave functions $\psi(\boldsymbol{r})=\varphi_{x v l m}(\boldsymbol{r})$ and the continuous spectrum wave functions $\psi(\boldsymbol{r})=\psi_{\boldsymbol{K}}^{ \pm}(\boldsymbol{r})$ are orthogonal to each other.

Note that the wave functions of the discrete $(E<0)$ and continuous $(E>0)$ spectra are solutions of the Sturm-Liouville problem based on the non-relativistic single-particle Schrödinger equation:

$$
\left[-\frac{\hbar^{2}}{2 M} \Delta+V_{A x}(r)\right] \Psi(\boldsymbol{r})=E \Psi(\boldsymbol{r}) .
$$


The bound state solutions of Equation (5) must satisfy the following integrability conditions:

$$
\left.r^{2+\delta}|\Psi(\boldsymbol{r})|^{2}\right|_{r \rightarrow \infty} \rightarrow 0, \delta>0 ;
$$

in the latter case, the condition (6) must be replaced by following condition:

$$
\begin{aligned}
& \Psi(\boldsymbol{r})=\left.\psi_{K}^{( \pm)}(\boldsymbol{r})\right|_{r \rightarrow \infty} \rightarrow \mathrm{e}^{i K r}+f\left(\boldsymbol{n}_{K}\right) \frac{\mathrm{e}^{ \pm i K r}}{r}, \\
& \boldsymbol{n}_{K}=\frac{\boldsymbol{K}}{|\boldsymbol{K}|} .
\end{aligned}
$$

One can recall that the vector $\boldsymbol{K}$ appearing in Equation (7) is the wave vector of the knocked-out nucleon: $K=|\boldsymbol{K}|=\sqrt{2 M E}$.

\section{The Cross Sections of the Nucleus Electro-Disintegration}

In this paper we restrict ourselves to those processes in which the knockout of protons and neutrons from the nuclei is associated with relatively small transfers of energy $\omega$ and momentum $|\boldsymbol{q}|$ from the scattered electrons to the atomic nucleus $A: \frac{\omega}{M} \ll 1 ; \frac{q}{M} \ll 1$; $\frac{K_{x}}{M} \ll 1$; In the present approximation the interaction of a relativistic electron with a non-point nucleon of the nucleus can be quite accurately described the quasi-relativistic Hamiltonian of McVoy-Van Hove [1,2], which takes into account empirically the electromagnetic structure of the knocking-out non-point nucleon in the form of relativistic corrections. After carrying out simple calculations (perturbation theory, the impulse approximation), the cross section $\sigma_{x v l}\left(\boldsymbol{k}, \boldsymbol{k}^{\prime}, \boldsymbol{K}\right)$ of knocking out of an $x$-nucleon from the $x v l$-shell of the nucleus $A$ by an inelastically scattered ultra-relativistic electron is presented in the following factorized expression $[5,7,21,25]$ :

$$
\begin{aligned}
& \sigma_{x v l}\left(\boldsymbol{k}, \boldsymbol{k}^{\prime}, \boldsymbol{K}\right) \equiv \frac{\mathrm{d}^{5} \sigma_{x v l}}{\mathrm{~d} \varepsilon^{\prime} \mathrm{d} \Omega^{\prime} \mathrm{d} \Omega} \\
& =e^{4} N_{x v l} F_{x}^{2}\left(q_{\mu}^{2}\right) \frac{4 M K}{\boldsymbol{k}^{2}} \times P\left(\boldsymbol{k}, \boldsymbol{k}^{\prime}\right) S_{x}\left(\boldsymbol{k}, \boldsymbol{k}^{\prime}, \boldsymbol{K}\right) G_{x v l}(\boldsymbol{q}, \boldsymbol{K}),
\end{aligned}
$$

where $e$ is the proton charge, $N_{x v l}$ is the number of $x$-nucleons in the nuclear $x v l$-shell,

$$
\begin{gathered}
\theta^{\prime} \equiv \theta_{\mathbf{k}^{\prime}}, \theta \equiv \theta_{\mathbf{K}}, \theta_{\mathbf{k}^{\prime}}=0, \varphi^{\prime} \equiv \varphi_{\mathbf{k}^{\prime}}=0, \varphi_{\mathbf{q}}=\pi, \\
\gamma_{x}=1.79 \delta_{x p}-1.91 \delta_{x n}, \\
F_{x}\left(q_{\mu}^{2}\right) \equiv F\left(q_{\mu}^{2}\right)=\left(1+0.055(F m)^{2} q_{\mu}^{2}\right)^{-2} ;
\end{gathered}
$$

$$
\begin{aligned}
S_{x}\left(\boldsymbol{k}, \boldsymbol{k}^{\prime}, \boldsymbol{K}\right) & =\frac{1}{2 k k^{\prime}}\left\{\delta _ { x p } \left[\left(1+\frac{\omega}{M}+\frac{\boldsymbol{q}^{2}\left(1-2 \gamma_{x}\right)}{4 M^{2}}\right)\left(\varepsilon \varepsilon^{\prime}+\boldsymbol{k} \boldsymbol{k}^{\prime}\right)\right.\right. \\
- & \left.\left.\frac{2}{M} \boldsymbol{K}\left(\varepsilon \boldsymbol{k}^{\prime}+\varepsilon^{\prime} \boldsymbol{k}\right)+\frac{q_{\mu}^{2} \boldsymbol{K}^{2}+4(\boldsymbol{k} \boldsymbol{K})\left(\boldsymbol{k}^{\prime} \boldsymbol{K}\right)}{2 M^{2}}\right]\right](12) \\
+ & \left.\gamma_{x}^{2} \frac{4\left[\boldsymbol{k} \boldsymbol{k}^{\prime}\right]^{2}+\left(q_{\mu}^{2}\right)^{2}}{4 M^{2}}\right\}
\end{aligned}
$$

is a dimensionless, positive definite and continuous $\left(\left|S_{x}\left(\boldsymbol{k}, \boldsymbol{k}^{\prime}, \boldsymbol{K}\right)\right| \sim 1\right)$ function of the vector arguments $\boldsymbol{k}, \boldsymbol{k}^{\prime}, \boldsymbol{K}$. It is in this function that the structural features of the interaction of the scattered relativistic electron and the knocked-out nucleon in the quasi-relativistic Hamiltonian of McVoy-Van Hove are reflected. Two essentially different functions appearing in Equation (8), which depend on kinematic variables of the process of the electro-disintegration of nuclei,

$$
P\left(\boldsymbol{k}, \boldsymbol{k}^{\prime}\right) \equiv P\left(\theta^{\prime}\right)=\frac{\boldsymbol{k}^{2} \boldsymbol{k}^{\prime 2}}{\left(q_{\mu}^{2}\right)^{2}}
$$

and

$$
\begin{aligned}
& G_{x v l}(\mathbf{q}, \mathbf{K})=\frac{1}{(2 l+1)(2 \pi)^{3}} \times \\
& \sum_{m=-l}^{m=l}\left|\int\left(\psi_{\mathbf{K}}^{(-)^{*}}(\mathbf{r}) \exp (i \mathbf{q r}) \varphi_{x v l m}(\mathbf{r})\right) \mathrm{d}^{3} \mathbf{r}\right|^{2},
\end{aligned}
$$

exert most comprehensive and determinative $[25,27]$ influence on the interpretation of various aspects of the processes electro-disintegration of nuclei. The function $G_{x v l}(\boldsymbol{q}, \boldsymbol{K})$ depending on the kinematic parameters $\boldsymbol{q}$ and $\boldsymbol{K}$ defines the distorted momentum distribution of nucleons in the $x v l$-shell of the atomic nucleus. It is $G_{x v l}(\boldsymbol{q}, \boldsymbol{K})$ that contains the most complete information about the dynamics of the nucleon in the nucleus force field $V_{A x}(r): \varphi_{x l m}(\boldsymbol{r})$ appearing in Equation (14) are the wave function of the nucleon in the bound state; $\psi_{\boldsymbol{K}_{x}}^{(-)}(\boldsymbol{r})$ is the wave function of the nucleon in the state of continuous spectrum.

If we take into account the influence of the nuclear field on the motion of the knocked-on nucleon in the final state, the factorization of expression (8) for the cross section $\sigma_{x v l}\left(\boldsymbol{k}, \boldsymbol{k}^{\prime}, \boldsymbol{K}\right)$ is approximate. The factorized expression (8) becomes exact only in the plane-wave approximation, which is valid when the energy of the knocking-out protons is quite large. In this case $\psi_{K}^{( \pm)}(\boldsymbol{r}) \approx \exp (i \boldsymbol{K} \boldsymbol{r})$, and $G_{x v l}(\boldsymbol{q}, \boldsymbol{K})$ transforms into $G_{x v l}^{0}(\boldsymbol{q}-\boldsymbol{K})$, which determines the momentum distribu- 
tion of the nucleons in the $x v l$-shell:

$$
\begin{aligned}
G_{x v l}^{0}(\mathbf{q}-\mathbf{K}) & =\frac{1}{(2 l+1)(2 \pi)^{3}} \\
& \cdot \sum_{m=-l}^{m=l}\left|\int \varphi_{x v l m}(\mathbf{x}) \exp (i(\mathbf{q}-\mathbf{K}) \mathbf{x}) \mathrm{d}^{3} \mathbf{x}\right|^{2} .
\end{aligned}
$$

Here and below the exclusive and inclusive cross sections of the nucleus electro-disintegration calculated in the plane-wave approximation will be labeled, if necessary, in the graphs, tables and formulas by the symbol 0 , and the similar values calculated with taking into account the interaction of the knocked-out nucleon in the final state will be labeled by the symbol d. Formula (8) specifies the initial exclusive cross section of electro-disintegration of the atomic nucleus. Experimental verification of (8) requires fairly laborious experiments in which both the inelastically scattered electron and the knocked-out proton are simultaneously registered or identified. At the moment, a large number of less laborious experiments are realized. In these experiments one investigates the energy distributions of inelastically scattered electrons at certain scattering angles and initial energies of the scattered electrons.

There are carefully developed methodologies taking into account the inelastically scattered electrons that have lost their energy in a variety of quantum-electro-dynamical $[28,30]$ processes such as bremsstrahlung, birth of electron-positron pairs, etc. If we subtract these electrons from other scattered ones, we obtain the inclusive cross section $\sigma\left(\omega, \theta^{\prime}\right)$ of inelastic scattering of highenergy electrons in the process of collisions of ultrarelativistic electrons and nuclei:

$$
\sigma\left(\omega, \theta^{\prime}\right)=\sum_{x v l} \sigma_{x v l}\left(\omega, \theta^{\prime}\right)=\sum_{x v l} N_{x v l} \sigma_{x v l}^{u}\left(\omega, \theta^{\prime}\right),
$$

where $N_{x v l}$ is the number of $x$-nucleons in $x v l$-nuclear shell, $\quad \sigma_{x v l}\left(\omega, \theta^{\prime}\right)$ and $\sigma_{x v l}^{u}\left(\omega, \theta^{\prime}\right)=\sigma_{x v l}\left(\omega, \theta^{\prime}\right) / N_{x v l}$ are the complete and specific knock-out cross sections of a $x$-nucleon from the $x v l$-shell of the atomic nucleus, respectively. The summation in Equation (9) is applied to all, fully or partially, filled shells of the investigated nucleus, and $\sigma_{x v l}\left(\omega, \theta^{\prime}\right)$ is calculated in 0 - and $d$-approximations by direct numerical integration of the differential cross section (8) over the total solid angle $\Omega \equiv \Omega_{K}$ of propagation of the knocked-out nucleons:

$$
\sigma_{x v l}\left(\omega, \theta^{\prime}\right) \equiv \frac{\mathrm{d}^{3} \sigma_{x v l}}{\mathrm{~d} \varepsilon^{\prime} \mathrm{d} \Omega^{\prime}}=\iint_{\Omega} \sigma_{x v l}\left(\mathbf{k}, \mathbf{k}^{\prime}, \mathbf{K}\right) \mathrm{d} \Omega_{\mathbf{K}} .
$$

Studying the reaction $A\left(e, e^{\prime} n\right)(A-1)$, we can extract realistic information about the structure of atomic nuclei in the framework of an investigated model by comparing the calculated and measured exclusive (8) and inclusive (17), (17) differential cross sections of the electro-disintegration of nuclei. If we restrict ourselves to the nuclear shell model then the calculations of these cross sections are associated with labor-intensive computing of the overlap integrals:

$$
\begin{aligned}
& I_{x v l m}\left(\boldsymbol{q}, \boldsymbol{K}_{x}\right) \equiv\left\langle\boldsymbol{K}_{x}\left|\mathrm{e}^{(i q r)}\right| x v l m\right\rangle \\
& =\iiint_{-\infty} \psi_{\boldsymbol{K}_{x}}^{(-)^{*}}(\boldsymbol{r})[\exp (i \boldsymbol{q} \boldsymbol{r})] \varphi_{x v l m}(\boldsymbol{r}) \mathrm{d}^{3} \boldsymbol{r} .
\end{aligned}
$$

These integrals determine the distorted momentum distributions (Equation (14)) of nucleons in the filled $x v l$-shells of atomic nuclei. The deductions and conclusions of this paper depend strongly on the numerical values of the calculated electro-disintegration cross sections of various nuclei. Because of this, the reliability and correctness [33] of applied numerical methods of the electro-disintegration cross-section calculation will be discussed in Appendix A in detail.

Note that the electro-disintegration processes of nuclei are relatively easy interpreted in the approximation presented below by the quantum-electro-dynamical Feynman diagram of second order.

If we suppose that the range of nuclear forces is restricted then we can assert that the wave function of the knocked-out $(E>0)$ nucleon [29] has the asymptotic form given by Equation (7). The condition (7) mentioned above once more affirms that the knocked-out nucleon moving in the region of the residual nucleus $(A-1)$ scatter itself elastically on this one.

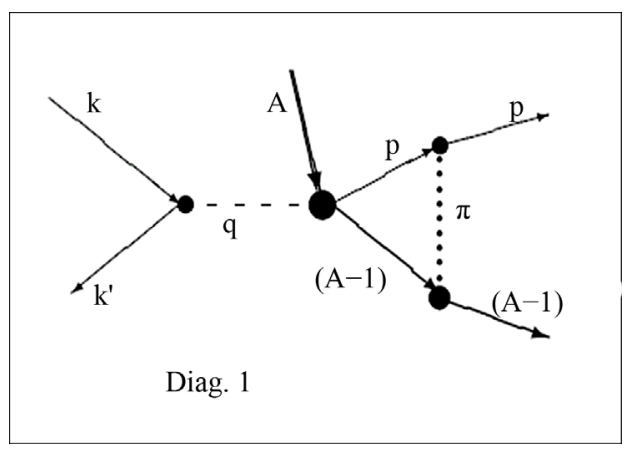

These processes of the nucleus electro-disintegration are presented on the above Feynman diagram. On this diagram, we can distinguish the initial electron with 4momentum $k \equiv k_{\mu}$, which acquires the status of a scattered electron $\left(k^{\prime} \equiv k_{\mu}^{\prime}\right)$ after emission of a virtual photon. The virtual photon $q$ is absorbed by one of the nucleons of the target nucleus and provokes the ejection of this nucleon from the nucleus. The knocked-out nucleon interacts with the residual nucleus in the final state. This interaction is presented on the diagram by a hypothetical exchange meson with 4-momentum $\pi$.

In this paper we estimate the dependence of the socalled $\chi$-sections (see Equation (35)) of nuclear electrodisintegration from the processes represented below by the sum of Feynman diagrams of higher order: 

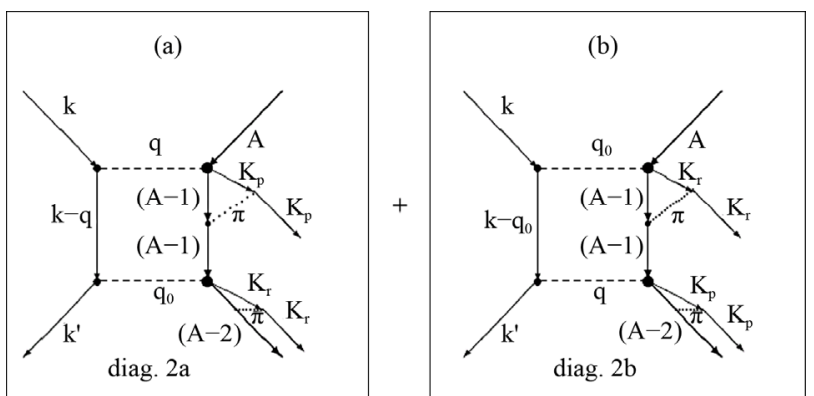

In these diagrams we see new additional participants of the more comprehensive theoretical interpretation of the inelastic scattering of high-energy electrons on nuclei. First and foremost, it is a quasi-real $\left(q_{0} \equiv q_{0 \mu}\right)$ virtual photon [25], which is absorbed by a proton of the nucleus $A$ and provokes the transition of this proton in the quasi-discrete state $K_{r}$ of the nucleus $A$ or $A-1$. The knocked-out protons $K_{p}$ and $K_{r}$ interact in the final state with the residual nucleus. This interaction is shown schematically in the diagrams by 4-momenta of $\pi=\pi_{\mu}$ of hypothetical mesons. The knocked-out nucleons exchange by such a meson with the daughter nucleus $A-2$. The Feynman diagrams (diag.2a, diag.2b) describe the processes of the two-proton knockout from the atomic nucleus, which are predicted by the nuclear shell model. As we will see later, these processes are also capable to provide a non-trivial impact on the results of experimental studies of reactions $A\left(e, e^{\prime} p\right)(A-1)$.

It is well known that 4-vertex quantum-electro-dynamical processes (diag.2a, diag.2b) in general case (if we substitute $q_{0} \rightarrow q^{\prime} \neq q_{0}, q \neq q_{0}$ in diag.2a, diag.2b) are weakly visible against the backgrounds of the 2vertex processes (diag.1). It is useful to remind once again that 4-vertex quantum-electro-dynamical processes (diag.2a, diag.2b) become apparent only in the case when in both diagrams (diag.2a and diag.2b) and in, at least, one of two left vertex of each diagram the electron emits a quasi-real photon $q_{0}\left(\theta^{\prime}=0\right.$, , $0-0$ "-scattering $)$. It is the quasi-real photon $q_{0}$ that, as we will see subsequently, is capable to excite with high probability the Coulomb resonance in the atomic nucleus.

It is necessary to note that the nucleon knocked out from the nucleus $A$ can participate in the processes of inelastic scattering on the residual $(A-1)$ nucleus. For example, this nucleon can spend a part of its energy for the excitation of the discrete state of the residual nucleus. Colliding with another nucleon of the residual nucleus, the knocked-out nucleon is capable to increase the number of knocked-out nucleons. Taking into account the process of inelastic scattering of the knocked-out nucleon on the residual nucleus can essentially complicate the interpretation of the electro-disintegration processes in the coincidence experiments.

\section{Quasi-Discrete Spectra of Atomic Nuclei}

Let us calculate the cross sections (8) and (16) of the knockout of a nucleon (reactions $A\left(e, e^{\prime} p\right)(A-1)$ ). In the nuclear shell model (LS-coupling) we approximate the potential $V_{A x}(r)$ by a sum of two terms: $V_{A x}(r)=V_{W S}(r)+\delta_{x p} V_{C}(r)$. The first term $V_{W S}(r)$ is the short-range Woods-Saxon potential:

$$
\begin{aligned}
& V_{W S}(r)=-\frac{V_{0 A x}}{1+\exp \left(\frac{r-R}{a}\right)} \approx-\frac{V_{0 A x} \Theta(b-r)}{1+\exp \left(\frac{r-R}{a}\right),} \\
& \left(V_{0 A x} \equiv V_{0 x}>0\right),
\end{aligned}
$$

where:

$$
\Theta(x)=\left(1-\delta_{x 0}\right) \frac{x+|x|}{2 x}+\frac{1}{2} \delta_{x 0}
$$

is the Heaviside unit function; $b \approx r_{0} \cdot \sqrt[3]{A}+20 a ; V_{0 A x}$, $a$ and $R=r_{o} \times \sqrt[3]{A}$ are parameters of the WoodsSaxon potential.

The second term $\delta_{x p} V_{C}(r)$ is the long-range Coulomb potential:

$$
\begin{aligned}
& V_{C}(r) \\
= & {\left[\frac{(Z-1) e^{2}}{R}\left(\frac{3}{2}-\frac{r^{2}}{2 R^{2}}\right) \Theta(R-r)+\frac{(Z-1) e^{2}}{r} \Theta(r-R)\right] . }
\end{aligned}
$$

The wave functions of continuous spectrum can be obtained in the form of the following series:

$$
\begin{aligned}
\psi_{K}^{( \pm)}(\boldsymbol{r}) & =4 \pi \sum_{l_{1}=0}^{l_{1}=\infty} \sum_{m_{1}=-l_{1}}^{m_{1}=l_{1}} i^{l_{1}} R_{K l_{1}}^{( \pm)}(r) Y_{l_{1} m_{1}}^{*}\left(\boldsymbol{n}_{r}\right) Y_{l_{1} m_{1}}\left(\boldsymbol{n}_{K}\right) . \\
= & 4 \pi \sum_{l_{1}=0}^{l_{1}=\infty} \sum_{m_{1}=-l_{1}}^{m_{1}=l_{1}} i_{1}^{l_{1}} A_{K l_{1}}^{( \pm)} \frac{Z_{K l_{1}}(r)}{r} Y_{l_{1} m_{1}}^{*}\left(\boldsymbol{n}_{r}\right) Y_{l_{1} m_{1}}\left(\boldsymbol{n}_{K}\right)
\end{aligned}
$$

The radial functions $Z_{\lambda}(r)=A_{K l} Z_{K l}(r),(\forall E>0)$. in the half-open space $0 \leq r<\infty$ in our model are always real and bounded solutions of the radial Schrödinger wave equation with the real potential $V_{A x}(r)$ :

$$
\frac{\mathrm{d}^{2} Z_{\lambda}(r)}{\mathrm{d} r^{2}}+\left[2 m\left(E-V_{A x}(r)\right)-\frac{l(l+1)}{r^{2}}\right] Z_{\lambda}(r)=0 .
$$

It is useful to remind [31] that the asymptotic behavior of the wave functions of continuous spectrum (7) determines the asymptotic behavior of the radial functions $Z_{\lambda}(r)$ :

$$
\begin{aligned}
Z_{\lambda}^{(-)}(r)= & \left\{\begin{array}{l}
A_{K l}^{(-)} Z_{K l}(r) \forall(r \leq b) ; \\
\frac{S_{K l}^{(-)}\left(\left(g_{l}(\rho)+i f_{l}(\rho)\right)-\left(g_{l}(\rho)-i f_{l}(\rho)\right)\right)}{2 i}(25)
\end{array}\right. \\
& \cdot \forall(r \geq b ; \rho=K r)
\end{aligned}
$$


Here: $g_{l}(\rho)$ and $f_{l}(\rho)(\rho=K r)$ in Equation (25) are the well-known [34,35] Coulomb functions $\left(V_{C}(r) \neq 0\right)$ :

$$
g_{l}(\rho)=G_{l}(\eta, \rho), f_{l}(\rho)=F_{l}(\eta, \rho),
$$

where

$$
\eta=(Z-1) e^{2} \sqrt{\frac{M}{2 E}}
$$

is the Coulomb parameter. If $V_{C}(r)=0$, then

$$
\begin{aligned}
& g_{l}(\rho)=-\rho n_{l}(\rho)=\lim _{\eta \rightarrow 0} G_{l}(\eta, \rho) ; \\
& f_{l}(\rho)=\rho j_{l}(\rho)=\lim _{\eta \rightarrow 0} F_{l}(\eta, \rho),
\end{aligned}
$$

where $n_{l}(\rho)$ and $j_{l}(\rho)$ are the Neumann and Bessel spherical functions.

Tailoring $[12,25,27]$ the solutions (25) of Equation (24) at the point $r=b$, we find the coefficients $A_{K L}^{(-)} \equiv A_{L}^{(-)}(E)$ :

$$
A_{L}^{(-)}(E)=\left[w_{G Z}^{L}(E)+i w_{F Z}^{L}(E)\right]^{-1},
$$

where

$$
\begin{gathered}
w_{G Z}^{L}(E)=\left.\left(G_{l}(\eta, \rho) \frac{\mathrm{d} Z_{K l}(r)}{\mathrm{d} r}-Z_{K l}(r) \frac{\mathrm{d} G_{l}(\eta, \rho)}{\mathrm{d} r}\right)\right|_{\substack{r=b \\
(29)}} ; \\
w_{F Z}^{L}(E)=\left.\left(F_{l}(\eta, \rho) \frac{\mathrm{d} Z_{K l}(r)}{\mathrm{d} r}-Z_{K l}(r) \frac{\mathrm{d} F_{l}(\eta, \rho)}{\mathrm{d} r}\right)\right|_{r=b} .
\end{gathered}
$$

Simple mathematical analysis of the amplitude expression $A_{l}^{(-)}(E)$ in Equation (28) [12] leads to the following conclusions. One can determine the energies of the Coulomb resonances $E=E_{p N L}^{r}$ from the condition [12]

$$
\left.\frac{\left(\frac{\mathrm{d} Z_{K L}(r)}{\mathrm{d} r}\right)}{Z_{K L}(r)}\right|_{r=b}=\left.\frac{\left(\frac{\mathrm{d} G_{L}(\eta, \rho)}{\mathrm{d} r}\right)}{G_{L}(\eta, \rho)}\right|_{r=b}
$$

and the half-width $\gamma_{N L p}^{r}$ from the equality [12]

$$
\frac{\gamma_{p N L}^{r}}{2}=\left.w_{F Z}^{L}(E)\left[\frac{\mathrm{d}\left(w_{G Z}^{L}(E)\right)}{\mathrm{d} E}\right]^{-1}\right|_{E=E_{N L}^{r}} .
$$

Direct mathematical analysis of the expression (28) for the amplitude $A_{L}^{(-)}(E)$ of the separate radial component $R_{K l}(r)$ reveals $[12,25,27]$ the cause of the abrupt increase of the magnitude of the cross section $\sigma\left(\omega, \theta^{\prime}\right)$ in the vicinity of the proton resonance energy $E=E_{p N L}^{r}$. We recall that the equalities (31) and (32) are true in the case when the strong inequality
$G_{L}^{2}(\eta, \rho)+\left(\frac{\mathrm{d} G_{L}(\eta, \rho)}{\mathrm{d} \rho}\right)^{2} \gg \gg F_{L}^{2}(\eta, \rho)+\left(\frac{\mathrm{d} F_{L}(\eta, \rho)}{\mathrm{d} \rho}\right)^{2}$

is valid. When the energy $E=E_{p N L}^{r}$ of Coulomb resonance approaches the Coulomb barrier height

$\left(E_{p N L}^{r} \geq \frac{2 V_{C}(b)}{3}\right)$, the mentioned strong inequality re-

laxes to a more delicate inequality:

$$
G_{L}^{2}(\eta, \rho)+\left(\frac{\mathrm{d} G_{L}(\eta, \rho)}{\mathrm{d} \rho}\right)^{2} \gg F_{L}^{2}(\eta, \rho)+\left(\frac{\mathrm{d} F_{L}(\eta, \rho)}{\mathrm{d} \rho}\right)^{2} .
$$

In that case the Coulomb resonances acquire the status of incipient resonances. Such Coulomb resonances become apparent when one calculates spectra of inelastically scattered electrons. In this case the peaks of resonances have moderate heights and rather large half-widths $\gamma_{p v l}^{r}$.

As an example of application of Equations (24)-(32), we present the calculated main characteristics (the energies $E_{N L x}$, the half-widths $\gamma_{N L x}$ and the wave function amplitudes $\left|A_{N L x}\right|$ ) of quasi-discrete levels of some atomic nuclei in Table 1. It is important to keep in mind that these characteristics can depend essentially [12,27] on the parameters of the Woods-Saxon potential. Notice that the parameters $r_{0}$ and $a$ of the Woods-Saxon potential in the calculations of this article are kept invariable: $r_{0}=1.24 \mathrm{Fm}$ and $a=0.55 \mathrm{Fm}$. This simplification does not influence the conclusions of this paper.

Note also that the analysis of the quasi-discrete spectra (jj-coupling) of the light, medium and heavy nuclei permits to affirm that the nucleus quasi-discrete spectrum is the natural extension of the nuclear shell structure to the continuous spectrum region. For this reason, the experimental and theoretical investigations of quasi-discrete spectra properties in $A\left(e, e^{\prime} p\right)(A-1)$-knockout reactions of protons are, at the same time, investigations of the nuclear shell model.

\section{The Cross-Sections of Excitation of Coulomb Resonances}

It is well known $[12,13,19,25,27]$ that theoretical investigations of the inelastic scattering of high-energy electrons predict the resonance structure of inclusive (Equation (9)) cross-sections as functions of transmitted energy $\omega(\omega \leq 50 \mathrm{MeV})$ in the framework of the nuclear shell model. The dynamic characteristics (height, half-width) of resonance peaks substantially changed due to the small variations of the parameters of Woods-Saxon potential. For instance, the maximum values of $\sigma\left(\omega_{v l \rightarrow N L}, \theta^{\prime}\right)$ and the half-widths $\gamma_{v l \rightarrow N L}$ of resonance cross-sections $\sigma\left(\omega_{v l \rightarrow N L}, \theta^{\prime}\right)$ can undergo enormous quantitative changes. Such huge variations of characteristics of resonance peaks undoubtedly require additional investigations of this phenomenon. 
Table 1. The principal physical characteristics of the quasi-discrete spectra of some atomic nuclei.

\begin{tabular}{|c|c|c|c|c|c|c|c|c|c|}
\hline$N L$ & $x$ & $E_{N L x}$ & $\left|A_{N L L}\right|$ & $\gamma_{N L x}$ & $N L$ & $x$ & $E_{N L x}$ & $\left|A_{N L x}\right|$ & $\gamma_{N L x}$ \\
\hline \multicolumn{5}{|c|}{${ }^{56} \mathrm{Fe} ; \quad V_{(0 p ; 0 n)}=(63.3 ; 50.55) \mathrm{MeV}$} & \multicolumn{5}{|c|}{${ }^{58} \mathrm{Ni} ; \quad V_{(0 p ; 0 n)}=(62.0 ; 49.3415) \mathrm{MeV}$} \\
\hline 14 & $\mathrm{~N}$ & $1.36 \times 10^{-2}$ & $3.82 \times 10^{5}$ & $3.05 \times 10^{-12}$ & 13 & $\mathrm{n}$ & $2.02 \times 10^{-3}$ & $1.67 \times 10^{7}$ & $6.06 \times 10^{-16}$ \\
\hline 14 & $\mathrm{P}$ & $1.36 \times 10^{-2}$ & $6.85 \times 10^{44}$ & $1.07 \times 10^{-90}$ & 14 & $\mathrm{p}$ & $5.97 \times 10^{-1}$ & $7.15 \times 10^{6}$ & $6.33 \times 10^{-14}$ \\
\hline 22 & $\mathrm{P}$ & 2.78 & 15.43 & $1.48 \times 10^{-2}$ & 15 & $\mathrm{p}$ & 3.35 & 10.76 & 0.47 \\
\hline \multicolumn{5}{|c|}{${ }^{81} \mathrm{Y} ; \quad V_{(0 p ; 0 n)}=(54.13 ; 41.734) \mathrm{MeV}$} & \multicolumn{5}{|c|}{${ }^{119} \mathrm{Sn} ; \quad V_{(0 p ; 0 n)}=(63.3 ; 50.55) \mathrm{MeV}$} \\
\hline 14 & $\mathrm{P}$ & $8.83 \times 10^{-3}$ & $3.27 \times 10^{78}$ & $3.51 \times 10^{-158}$ & 23 & $\mathrm{p}$ & 2.26 & $6.0932 \times 10^{3}$ & $1.06 \times 10^{-7}$ \\
\hline 22 & $\mathrm{~N}$ & $4.40 \times 10^{-2}$ & 31.58 & $3.15 \times 10^{-4}$ & 31 & $\mathrm{p}$ & 3.96 & $2.6415 \times 10^{1}$ & $5.09 \times 10^{-3}$ \\
\hline 22 & $\mathrm{P}$ & 3.48 & 22.43 & $8.17 \times 10^{-3}$ & 16 & $\mathrm{p}$ & 7.20 & $2.4490 \times 10^{2}$ & $1.82 \times 10^{-4}$ \\
\hline 30 & $\mathrm{P}$ & 3.99 & 4.12 & $1.60 \times 10^{-1}$ & 17 & $\mathrm{n}$ & 11.48 & 5.96 & $3.34 \times 10^{-1}$ \\
\hline 15 & $\mathrm{P}$ & 10.31 & 11.18 & $9.52 \times 10^{-2}$ & 17 & $\mathrm{p}$ & 17.64 & 9.98 & $1.60 \times 10^{-1}$ \\
\hline \multicolumn{5}{|c|}{${ }^{27} \mathrm{Al} ; \quad V_{(0 p ; 0 n)}=(63.5 ; 54.0) \mathrm{MeV}$} & \multicolumn{5}{|c|}{${ }^{40} \mathrm{Ca} ; \quad V_{(0 p ; 0 n)}=(53.9 ; 42.352) \mathrm{MeV}$} \\
\hline 13 & $\mathrm{P}$ & 0.455 & $9.17 \times 10^{3}$ & $3.3172 \times 10^{-8}$ & 13 & $\mathrm{n}$ & $5.27 \times 10^{-3}$ & $3.79 \times 10^{4}$ & $1.76 \times 10^{-10}$ \\
\hline 13 & $\mathrm{~N}$ & 0.748 & 31.20 & $3.2143 \times 10^{-3}$ & 13 & $\mathrm{p}$ & $6.06 \times 10^{-3}$ & $3.93 \times 10^{51}$ & $2.02 \times 10^{-104}$ \\
\hline 21 & $\mathrm{P}$ & 0.802 & 22.63 & $3.0319 \times 10^{-3}$ & 21 & $\mathrm{p}$ & 1.56 & $1.77 \times 10^{1}$ & $7.54 \times 10^{-3}$ \\
\hline \multicolumn{5}{|c|}{${ }^{208} \mathrm{~Pb} ; \quad V_{(0 p ; 0 n)}=(57.48 ; 47.4125) \mathrm{MeV}$} & \multicolumn{5}{|c|}{${ }^{198} \mathrm{Au} ; \quad V_{(0 p ; 0 n)}=(57.48 ; 47.4125) \mathrm{MeV}$} \\
\hline 17 & $\mathrm{~N}$ & $8.17 \times 10^{-3}$ & $9.71 \times 10^{10}$ & $3.57 \times 10^{-23}$ & 17 & - & - & - & - \\
\hline 23 & $\mathrm{P}$ & $2.70 \times 10^{-2}$ & $5.75 \times 10^{100}$ & $1.31 \times 10^{-202}$ & 23 & $\mathrm{p}$ & $6.72 \times 10^{-1}$ & $3.09 \times 10^{15}$ & $2.28 \times 10^{-31}$ \\
\hline 16 & $\mathrm{P}$ & 1.98 & $1.30 \times 10^{9}$ & $3.22 \times 10^{-18}$ & 17 & $\mathrm{n}$ & 1.39 & $1.88 \times 10^{3}$ & $1.25 \times 10^{-6}$ \\
\hline 31 & $\mathrm{P}$ & 2.39 & $4.36 \times 10^{5}$ & $1.66 \times 10^{-11}$ & 16 & $\mathrm{p}$ & 2.86 & $6.82 \times 10^{6}$ & $1.41 \times 10^{-13}$ \\
\hline 24 & $\mathrm{P}$ & 7.74 & 77.53 & $1.16 \times 10^{-3}$ & 31 & $\mathrm{p}$ & 2.99 & $1.90 \times 10^{4}$ & $9.65 \times 10^{-9}$ \\
\hline 18 & $\mathrm{~N}$ & 8.30 & 17.37 & $3.38 \times 10^{-2}$ & 24 & $\mathrm{p}$ & 8.49 & $3.67 \times 10^{1}$ & $5.35 \times 10^{-3}$ \\
\hline 32 & $\mathrm{P}$ & 9.83 & 5.00 & $2.12 \times 10^{-1}$ & 18 & $\mathrm{n}$ & 9.81 & 11.14 & $8.78 \times 10^{-2}$ \\
\hline 17 & $\mathrm{P}$ & 10.06 & $3.71 \times 10^{2}$ & $8.92 \times 10^{-5}$ & 17 & $\mathrm{p}$ & 11.14 & $1.73 \times 10^{2}$ & $4.31 \times 10^{-4}$ \\
\hline 18 & $\mathrm{P}$ & 18.53 & 22.39 & $3.23 \times 10^{-2}$ & 18 & $\mathrm{p}$ & 19.79 & 15.88 & $6.67 \times 10^{-2}$ \\
\hline
\end{tabular}

Note that in this case the location of peaks $\omega=\omega_{v l \rightarrow N L}$ remains $[25,27]$ practically unchanged. Note once again that, for example, the microscopical modifications $\left(\leq 10^{-4}\right.$ $\mathrm{MeV}$ ) of depth $V_{0 p}$ of Woods-Saxon potential can lead to an increase of the Coulomb resonance peak $\left(\sigma\left(\omega_{v l \rightarrow N L}, \theta^{\prime}\right)\right)$ of order of $10^{100}-10^{200}$ times and a corresponding decrease of the half-width of this resonance of order of $10^{-100}-10^{-200}$ times. In this connection it is necessary to investigate the influence of variations of the nuclear shell potential parameters on the theoretically predicted measurable values of cross-sections of excitations of Coulomb and centrifugal resonances.

It is worth noting that the absence of inter-compensative relation between the increase of height of any peak and the decrease of its half-width would mean the existence of almost insurmountable difficulties in the interpretation of processes of nuclei electro-disintegration in the framework of the nuclear shell model. However, we will see that a correlation of high order between the increase (decrease) of the height of Coulomb resonance and the "adequate" decrease (increase) of the half-width of this resonance does exist in the cross section given by Equation(16) [27]. Indeed, the predicted values of the resonance cross sections $\sigma\left(\omega_{v l \rightarrow N L}, \theta^{\prime}\right)$ (which are measured in experiment) practically do not depend on the height and half-width of Coulomb resonances, as we will see below.

Let us consider an example of the influence of socalled quasi-real photons [25,27] on the scattering of high energy electrons on nuclei. Let us recall that the quasi-real photons are the result of such collision of a high energy electron and a nucleus when the direction of movement of the electron is practically unchanged after it ( $\theta^{\prime}=0$, “0-0" - scattering): $\frac{\boldsymbol{k}}{|\boldsymbol{k}|} \approx \frac{\boldsymbol{k}^{\prime}}{\left|\boldsymbol{k}^{\prime}\right|}$. The energy $\omega$ transmitted at such a frontal collision and the value of the transmitted momentum $|\boldsymbol{q}|=\left|\boldsymbol{k}-\boldsymbol{k}^{\prime}\right|$ (the energy and momentum of the quasi-real photon $q=q_{0}$ on the Feynman diagrams (the diag.2a and diag.2b)) are approximately equal. Let us recall also $[25,27]$ that the cross section $\sigma\left(\omega, \theta^{\prime}\right)$ as a function of the electron scattering angle $\theta^{\prime}$ have a sharp maximum at the point $\theta^{\prime}=0$, 
especially in the region where transmitted energy $\omega$ is not large $\left(\omega \ll \varepsilon^{\prime}\right)$. Moreover, our theory confirms [25, 27 that it is the quasi-real photons that gives the main contribution to the knockout of the protons in the reaction $A\left(e, e^{\prime} p\right)(A-1)$ in the investigated kinematic region. Scattering of electrons to large angles is barely noticeable in the proton knock-out mentioned above.

The theoretical interpretation of the inclusive crosssection $\sigma\left(\omega, \theta^{\prime}\right)$ as a function of $\omega$ allows us to confirm that at arbitrary electron scattering angle $\theta^{\prime}$ $\left(0 \leq \theta^{\prime} \leq \pi\right)$ and for some values of transmitted energy $\omega=\omega_{v l \rightarrow N L}$ one can see sharp and high $\left(10^{300} \mathrm{~b} / \mathrm{MeV} / \mathrm{sr}\right.$ and more) peaks on the plots of $\sigma\left(\omega, \theta^{\prime}\right)$. The halfwidth $\gamma_{v l \rightarrow N L}$ of such peaks may be abnormally small $\left(10^{-300} \mathrm{MeV}\right.$ and less). These peaks correspond to the excitation of so-called Coulomb $(x=p)$ and centrifugal $(x=n)$ resonances in atomic nuclei. Note that the excitation energy of the resonances is equal to the sum of the binding energy of the nucleon $\varepsilon_{x v l}$ in the nuclear $x v l$-shell and the energy of the Coulomb (centrifugal) resonance $E_{x N L}: \omega_{x v l \rightarrow x N L}^{r}=\left|\varepsilon_{x v l}\right|+E_{x N L}$.

Taking into account properties of the function $\sigma_{x v l}\left(\omega, \theta^{\prime}\right)$ and properties of the inelastic electron scattering with $\omega=\omega_{v l \rightarrow N L}$ and $\theta^{\prime} \approx 0$, one can conclude that the integral

$$
\begin{aligned}
& \sigma_{p v l \rightarrow p N L}^{r} \equiv N_{p v l} \times \sigma_{v l \rightarrow N L}^{u r}=\int_{\omega_{r}-\delta E}^{\omega_{r}+\delta E} \int_{0}^{\theta_{0}^{\prime}} \int_{0}^{2 \pi} \sigma\left(\omega, \theta^{\prime}\right) \mathrm{d} \omega \mathrm{d} \Omega^{\prime}, \\
& \delta E \gg \gamma_{N L}^{r},
\end{aligned}
$$

determines the lower limit of the complete $\left(\sigma_{p v l \rightarrow p N L}^{r}\right)$ and specific $\left(\sigma_{p v l \rightarrow p N L}^{u r}\right)$ cross-sections of excitation of the $p N L$-Coulomb resonance of the investigated atomic nucleus quite accurately. The upper limit of integration over the angle, $\theta^{\prime}=\theta_{0}^{\prime} \ll 1$, appearing in Equation (33) is a small quantity. As a rule, we restrict ourselves to the value of $\theta_{0} \approx \frac{\pi}{60}$ during the numerical integration.

The total cross-section of excitation of the Coulomb $N L$-resonance $\sigma_{N L}^{r}$ is equal to the sum

$$
\sigma_{N L}^{r}=\sum_{p v l} \sigma_{p v l \rightarrow N L}
$$

In the case of low Coulomb $N L$-resonances this crosssection is defined mainly by the only term corresponding to the dipole transition: $N_{L}=v_{l}, \quad L=l+1$.

For instance, let us consider the process of inelastic scattering of electrons with the energy $\varepsilon=500 \mathrm{MeV}$ on the nucleus ${ }^{40} \mathrm{Ca}$. The results for specific cross-sections $\sigma_{v l \rightarrow N L}^{u r}$ of excitation of the Coulomb resonances of the nucleus ${ }^{40} \mathrm{Ca}$ with the quantum numbers $N L=13,21$ are presented in Table 2 . The protons are knocked out from different occupied shells $(v l=10,11,12,20)$ of this nucleus. The dominant position in the excitation of
Table 2. The theoretical predictions of specific cross-sections $\sigma_{v l \rightarrow N L}^{u r}$ and energies $\omega_{v l \rightarrow N L}^{r}$ of excitation of $p N L$ Coulomb resonances with quantum numbers $N L=13.21$ in the nucleus ${ }^{40} \mathrm{Ca}$. The initial energy of the inelastically scattered electrons is $\varepsilon=500 \mathrm{MeV}$. The half-widths of that resonances are $\gamma_{13 p}^{r}=1.964 \times 10^{-117} \mathrm{MeV}$ and $\gamma_{21 p}^{r}=7.51$ $\times 10^{-3} \mathrm{MeV}$. The depth parameter of the Woods-Saxon potential is $V_{0 p}=53.9020 \mathrm{MeV}$.

\begin{tabular}{ccccc}
\hline$v l$ & $\sigma_{v l \rightarrow 13}^{r u}, \mathrm{~b}$ & $\omega_{v l \rightarrow 13}^{r}, \mathrm{MeV}$ & $\sigma_{v l \rightarrow 21}^{r u}, \mathrm{~b}$ & $\omega_{v l \rightarrow 21}^{r}, \mathrm{MeV}$ \\
\hline 10 & $2.288 \times 10^{-3}$ & 33.59 & $8.414 \times 10^{-3}$ & 35.54 \\
11 & $1.265 \times 10^{-1}$ & 23.59 & $9.12 \times 10^{-2}$ & 25.31 \\
12 & 15.19 & 12.20 & 2.19 & 13.96 \\
20 & $7.55 \times 10^{-5}$ & 9.57 & 17.98 & 11.29 \\
\hline
\end{tabular}

Coulomb resonances belongs to the dipole transitions: $[12] \rightarrow[13],[20] \rightarrow[21]$, as follows from the results given in Table 2.

In this case we assume that the radial quantum numbers are equal to each other: $v_{l}=N_{L}$. One can see that the excitation of Coulomb resonances caused by the quadrupole $([11] \rightarrow[13])$, octupole $([10] \rightarrow[13])$, etc. transitions is very difficult for observation against the background of dipole ones. We would like to mention, for example, that $\sigma_{[12] \rightarrow[13]}^{u r} \gg \sigma_{[10] \rightarrow[13]}^{u r}$ for the chosen parameters of the model. At the same time, it is possible that the above inequality may be strongly weakened or even violated for arbitrary $\theta^{\prime} \sim 1$.

Let us assume that the radial quantum numbers are not equal now: $v_{l} \neq N_{L}$ (usually, $N_{L}=v_{l}+1$ ). In this case the magnitude of $\sigma_{v l \rightarrow N L}^{u r}$ is significantly smaller as compared with the case of $v_{l}=N_{L}$.

The effect considered above may be a starting point for the interpretation of the phenomenon of gigantic dipole resonance in the framework of the nuclear shell model. Thus, the investigation of the inelastic scattering of high-energy electrons in the region where the transmitted energy is not large can be used for studying the phenomenon of gigantic dipole resonance. It is interesting to note that the above results have to some extent general character and common quantum nature.

These results do not depend on the choice of the nuclear target and the energy of the scattered ultra-relativistic electrons. For instance, let us consider the excitation of the Coulomb resonance with the quantum numbers $[N L]=[12]$ in the nucleus ${ }^{12} \mathrm{C}$ when a proton is knocked out from one of two filled shells $([v l]=[10],[11])$. The initial energy of the scattered electrons is $\mathcal{E}=2020$ $\mathrm{MeV}$. It follows from the data of Table 3 that the excitation cross-section $\sigma_{[p 11] \rightarrow[p 12]}^{r u}$ substantially exceeds the cross-section $\sigma_{[p 10] \rightarrow[p 12]}^{r u}$. Let us note that the cross sections of exitation of Coulomb resonances as well as the role of quasi-real photons increase significantly with an increase of the initial energee $\varepsilon$ of the scattered 
Table 3. Main physical characteristics (energies $E_{p 12}^{r}$, maximum amplitudes $\left|A_{p 12}^{r}\right|$, half-widths $\gamma_{p 12}^{r}$ ) of the Coulomb resonance $N L=12$, specific cross-sections $\sigma_{v l \rightarrow N L}^{r}$ and energies $\omega_{v l \rightarrow N L}$ of excitation of the Coulomb resonance $N L=[12]$ in the nucleus ${ }^{12} \mathrm{C}$ versus the depth $V_{0 p}$ of the Woods-Saxon potential. The initial energy of the inelastically scattered electrons is $\varepsilon=2020 \mathrm{MeV}$.

\begin{tabular}{cccccccc}
\hline$E_{p 12}^{r}, \mathrm{MeV}$ & $\left|A_{p 12}^{r}\right|$ & $\gamma_{p 12}^{r}, \mathrm{MeV}$ & $\sigma_{11 \rightarrow 12}^{r u}, \mathrm{~b}$ & $\sigma_{10 \rightarrow 12}^{r u}, \mathrm{~b}$ & $\omega_{11 \rightarrow 12}^{r}, \mathrm{MeV}$ & $V_{0 p} / \mathrm{MeV}$ \\
\hline 0.0062 & $6.85 \times 10^{13}$ & $6.36 \times 10^{-29}$ & 540.4 & 2.705 & 16.71 & 61.412 \\
0.182 & 750.8 & $2.818 \times 10^{-6}$ & 539.98 & 2.654 & 16.48 & 60.5 \\
2.090 & 5.23 & 0.169 & 570.2 & 2.52 & 15.24 & 56.0 \\
\hline
\end{tabular}

electrons [25,27]. We note also that the phenomenon of the anomalous increase of the scattering cross-section caused by quasi-real photons with an increase of the initial energy of the electron beam may have significant influence on the results of investigation of various aspects of nuclear structure. For instance, the results of experimental measurements of different cross-sections of inelastic electron scattering can essentially depend on the target thickness.

As an additional illustration of the above statements, in the Table 4 we present the calculated values of specific cross-sections of the Coulomb resonances excitation in the heavy nucleus ${ }^{198} \mathrm{Au}$ for two initial energies of scattered electrons: $\varepsilon=2020 \mathrm{MeV}$ and $\varepsilon=3365 \mathrm{MeV}$. The results of Table 4 do not require additional comments.

We would like to attract attention the fact that the inclusive cross-sections $\sigma\left(\omega, \theta^{\prime}\right)$ have the form of sharp resonance peaks at certain values of the transmitted energy $\omega=\omega_{v l \rightarrow N L}^{r}$. Note that the theoretically predicted

enormous heights and insignificantly small half-widths of $\sigma\left(\omega_{v l \rightarrow N L}^{r}, \theta^{\prime}\right)$ give no possibility to determine directly the shape of the Coulomb resonances in experiment. That is why we can determine only integral characteristics of the Coulomb resonances, such as the crosssections $\sigma_{p v l \rightarrow p N L}^{r}$ of excitation of these resonances, in the physical experiment.

Let us recall once again $[25,27]$ that the physical characteristics (the half-widths $\gamma_{N L p}^{r}$ and the amplitudes $\left(\left|A_{N L p}\right|\right)$ of the wave functions) of Coulomb resonances can undergo essential changes. For example, the sharp decrease of $\gamma_{N L p}^{r}$ and the equally drastic increase of $\left.\left|A_{N L p}\right|\right)$ is possible even at negligible changes of parameters of nuclear shell model potential. This uncertainty raises the question whether the cross-sections of excitation of Coulomb resonances undergo essential changes too. Table 5 gives an answer to this question.

In Table $\mathbf{5}$ we present the calculated functional dependence of the most important specific cross-sections $\sigma_{p v l \rightarrow p N L}^{r}$ of excitation of resonances of nucleus ${ }^{40} \mathrm{Ca}$. These cross-sections are interpreted as functions of the half-width of Coulomb resonances, which varies with the change of the depth $V_{0 p}$ of the Woods-Saxon potential. Note that the specific cross-sections mentioned above are caused by the dipole transitions [12] $\rightarrow[13],[20] \rightarrow[21]$ which, in turn, are caused by quasi-real photons in inelastic scattering of ultra-relativistic electrons on the nucleus ${ }^{40} \mathrm{Ca}$.

The results presented in the Table 5 do not require extensive comments as well. The specific cross-sections of the Coulomb resonance excitation $\sigma_{p v l \rightarrow p N L}^{r u}$ and the excitation energies $\omega_{v l \rightarrow N L}^{r}$ are practically invariant when the half-widths and the amplitudes of the wave function of Coulomb resonances change substantially. This result is very important. It essentially increases the chances of success for the nuclear shell model in the interpretation of inelastic electron scattering experiments aimed at studying the nuclear structure.

\section{The Inclusive Cross-Sections $\sigma\left(\omega, \theta^{\prime}\right)$ and the Coulomb Resonances}

One of the top-priority tasks of this paper is to study the manifestation of Coulomb and centrifugal resonances in experiments on inelastic scattering of high-energy electrons. In particular, it is of interest to investigate the possibilities to disclose and identify Coulomb and centrifugal resonances in the spectra of high-energy electrons inelastically scattered on various atomic nuclei.

Comparing the Coulomb resonances and the quasielastic peaks, one can state that the half-widths of the theoretically calculated peaks of Coulomb and centrifugal resonances are, as a rule, considerably less than the half-widths of the calculated quasi-elastic peaks. At the same time, the height of a Coulomb resonance peak is significantly larger than the height of a quasi-elastic peak. For convenience, such peaks of $\sigma\left(\omega, \theta^{\prime}\right)$ should be "cut off" on the plots of $\sigma\left(\omega, \theta^{\prime}\right)$ versus transmitted energy $\omega$. For this reason, we lose clarity and important information about the observability of Coulomb resonances in the measured spectra of inelastically scattered electrons. Taking the logarithm of that function adds very little information since the microscopical half-width of a Coulomb resonance can hardly be represented on the plot.

In order to investigate the possibility of detection of Coulomb resonances in physical experiment, one should recall that the cross-section $\sigma\left(\omega, \theta^{\prime}\right)$ at the arbitrary 
Table 4. Specific cross-sections $\sigma_{[v l] \rightarrow[N L]}^{r u}$ of excitation of the $p N L$-Coulomb resonances with the quantum numbers $N L$ in the nucleus ${ }^{198} \mathrm{Au}$. The initial energies of the inelastically scattered electrons are $\varepsilon=2020 \mathrm{MeV}$ and $\varepsilon=3595 \mathrm{MeV}$. The parameters of the Woods-Saxon potential are $V_{0 p}=51.13 \mathrm{MeV}$ and $V_{0 n}=41.734 \mathrm{MeV}$.

\begin{tabular}{|c|c|c|c|c|c|c|}
\hline \multicolumn{7}{|c|}{$\varepsilon=2020 \mathrm{MeV}$} \\
\hline$N L \rightarrow$ & 23 & 16 & 31 & 24 & 17 & 18 \\
\hline$v l \Downarrow$ & $\sigma_{v l \rightarrow 23}^{r u}, \mathrm{~b}$ & $\sigma_{v l \rightarrow 16}^{r u}, \mathrm{~b}$ & $\sigma_{v l \rightarrow 31}^{r u}, \mathrm{~b}$ & $\sigma_{v l \rightarrow 24}^{r u}, \mathrm{~b}$ & $\sigma_{v l \rightarrow 17}^{r u}, \mathrm{~b}$ & $\sigma_{v l \rightarrow 18}^{r u}, \mathrm{~b}$ \\
\hline 10 & $2.42 \times 10^{-2}$ & $1.28 \times 10^{-6}$ & $1.97 \times 10^{-2}$ & $1.89 \times 10^{-3}$ & $3.73 \times 10^{-6}$ & $1.45 \times 10^{-4}$ \\
\hline 11 & $2.57 \times 10^{-1}$ & $6.04 \times 10^{-5}$ & $3.67 \times 10^{-1}$ & $2.41 \times 10^{-2}$ & $5.97 \times 10^{-6}$ & $4.28 \times 10^{-5}$ \\
\hline 12 & 1.20 & $2.91 \times 10^{-3}$ & $3.00 \times 10^{-1}$ & $2.29 \times 10^{-1}$ & $2.12 \times 10^{-4}$ & $7.32 \times 10^{-4}$ \\
\hline 20 & $9.82 \times 10^{-2}$ & $8.81 \times 10^{-8}$ & 1.88 & $4.54 \times 10^{-3}$ & $8.49 \times 10^{-5}$ & $1.42 \times 10^{-3}$ \\
\hline 13 & 3.82 & $1.31 \times 10^{-1}$ & $3.10 \times 10^{-1}$ & $7.22 \times 10^{-1}$ & $6.50 \times 10^{-3}$ & $2.62 \times 10^{-3}$ \\
\hline 21 & 4.08 & $1.03 \times 10^{-6}$ & 5.71 & $1.16 \times 10^{-1}$ & $1.20 \times 10^{-4}$ & $3.59 \times 10^{-3}$ \\
\hline 14 & 45.3 & 6.73 & $3.19 \times 10^{-3}$ & 4.49 & $2.21 \times 10^{-1}$ & $1.49 \times 10^{-2}$ \\
\hline 22 & 498.0 & $1.04 \times 10^{-5}$ & 108.3 & 5.39 & $7.41 \times 10^{-4}$ & $7.67 \times 10^{-3}$ \\
\hline 30 & $1.81 \times 10^{-3}$ & $3.60 \times 10^{-12}$ & 596.3 & $1.23 \times 10^{-3}$ & $2.10 \times 10^{-3}$ & $9.15 \times 10^{-3}$ \\
\hline 15 & $2.02 \times 10^{-1}$ & 665.5 & $5.22 \times 10^{-7}$ & 43.53 & 8.89 & $3.57 \times 10^{-1}$ \\
\hline \multicolumn{7}{|c|}{$\varepsilon=3595 \mathrm{MeV}$} \\
\hline 10 & 0.077 & $4.07 \times 10^{-6}$ & $6.27 \times 10^{-2}$ & $6.02 \times 10^{-3}$ & $1.19 \times 10^{-5}$ & $4.66 \times 10^{-4}$ \\
\hline 11 & 0.817 & $1.92 \times 10^{-4}$ & 1.17 & $7.68 \times 10^{-2}$ & $1.91 \times 10^{-5}$ & $1.37 \times 10^{-4}$ \\
\hline 12 & 3.81 & $9.22 \times 10^{-3}$ & $9.52 \times 10^{-1}$ & $7.27 \times 10^{-1}$ & $6.75 \times 10^{-4}$ & $2.34 \times 10^{-3}$ \\
\hline 20 & 0.311 & $2.79 \times 10^{-7}$ & 5.95 & $1.44 \times 10^{-2}$ & $2.70 \times 10^{-4}$ & $4.53 \times 10^{-3}$ \\
\hline 13 & 12.1 & $4.15 \times 10^{-1}$ & $9.81 \times 10^{-1}$ & 2.29 & $2.06 \times 10^{-2}$ & $8.36 \times 10^{-3}$ \\
\hline 21 & 12.9 & $3.25 \times 10^{-6}$ & 18.0 & $3.69 \times 10^{-1}$ & $3.81 \times 10^{-4}$ & $1.14 \times 10^{-2}$ \\
\hline 14 & 143.0 & 21.2 & $1.00 \times 10^{-2}$ & 14.2 & $7.00 \times 10^{-1}$ & $4.75 \times 10^{-2}$ \\
\hline 22 & 1566.0 & $3.28 \times 10^{-5}$ & 341.1 & 17.0 & $2.34 \times 10^{-3}$ & $2.43 \times 10^{-2}$ \\
\hline 30 & $5.67 \times 10^{-3}$ & $1.13 \times 10^{-11}$ & 1876.0 & $3.87 \times 10^{-3}$ & $6.63 \times 10^{-3}$ & $2.90 \times 10^{-2}$ \\
\hline 15 & 0.636 & 2093.0 & $1.64 \times 10^{-6}$ & 137.3 & 28.1 & 1.13 \\
\hline
\end{tabular}

Table 5. Specific cross-sections $\sigma_{v l \rightarrow N L}^{r u}$ and energies $\omega_{v l \rightarrow N L}$ of excitation of the Coulomb resonances with the quantum numbers $[13,21]$ in the nucleus ${ }^{40} \mathrm{Ca}$ at inelastic scattering of electrons versus the resonance half-width $\gamma_{N L p}^{r}$. The initial energy of the electrons is $\varepsilon=500 \mathrm{MeV}$. The angle of scattering is $\theta^{\prime}==0$.

\begin{tabular}{ccccccc}
\hline$\sigma_{12 \rightarrow 13}^{r u}, \mathrm{~b}$ & $\omega_{12 \rightarrow 13}^{r}, \mathrm{MeV}$ & $\gamma_{13 p}^{r}, \mathrm{MeV}$ & $\sigma_{20 \rightarrow 21}^{r u}, \mathrm{~b}$ & $\omega_{20 \rightarrow 21}^{r}, \mathrm{MeV}$ & $\gamma_{21 p}^{r}, \mathrm{MeV}$ & $V_{0 p} / \mathrm{MeV}$ \\
\hline 15.19 & 12.20 & $1.116 \times 10^{-294}$ & 17.98 & 11.30 & $7.41 \times 10^{-3}$ & 53.9084 \\
15.19 & 12.20 & $4.688 \times 10^{-151}$ & 17.98 & 11.29 & $7.46 \times 10^{-3}$ & 53.9050 \\
15.19 & 12.20 & $1.964 \times 10^{-117}$ & 17.98 & 11.29 & $7.51 \times 10^{-3}$ & 53.9020 \\
15.20 & 12.19 & $2.935 \times 10^{-38}$ & 17.98 & 11.27 & $8.46 \times 10^{-3}$ & 53.8400 \\
15.22 & 12.18 & $6.105 \times 10^{-25}$ & 17.97 & 11.48 & $1.00 \times 10^{-2}$ & 53.7500 \\
15.31 & 12.10 & $3.392 \times 10^{-11}$ & 18.00 & 11.19 & $2.41 \times 10^{-2}$ & 53.2000 \\
15.52 & 11.87 & $3.680 \times 10^{-6}$ & 18.47 & 10.65 & $9.22 \times 10^{-2}$ & 52.0000 \\
15.60 & 11.87 & $2.917 \times 10^{-5}$ & 18.90 & 10.64 & $1.40 \times 10^{-1}$ & 51.5000 \\
\hline
\end{tabular}

point $\left(\omega, \theta^{\prime}\right)$ is measured experimentally by means of "averaging"" procedure:

$$
\sigma\left(\omega, \theta^{\prime}\right)=\frac{1}{2 \Delta E_{x} \cdot \Omega_{x}} \int_{\omega_{0}-\Delta E_{x} \Omega_{x}}^{\omega_{0}+\Delta E_{x}} \iint_{\varsigma}\left(\omega, \theta^{\prime}\right) \sigma\left(\omega, \theta^{\prime}\right) \mathrm{d} \omega \mathrm{d} \Omega^{\prime}
$$

Here $2 \Delta E_{x}$ is the spectral width of gap of the scattered electron spectrometer. Note that $\varsigma\left(\omega, \theta^{\prime}\right)$ is the function that determines the efficiency of registration of the scattered electrons at various angles of scattering $\theta^{\prime}$ and energies $\varepsilon^{\prime}=\varepsilon-\omega$. Hereinafter we suppose that $\varsigma\left(\omega, \theta^{\prime}\right)=1$. Further on in this paper we assume that the 
strong inequality $\gamma_{N L x} \ll 2 \Delta E_{x}$ is true.

It is worth noting that this inequality may be broken in the case of medium and heavy nuclei in our model. This circumstance is connected with the existence [27] of the so-called incipient $\left(0.5 \mathrm{MeV} \leq \gamma_{N L x} \leq 2 \mathrm{MeV}\right)$ Coulomb resonances. When they take place, it is quite possible that the inequality $\gamma_{N L x} \approx 2 \Delta E_{x}$ mentioned above may be broken and even inverted: $\gamma_{N L x} \geq 2 \Delta E_{x}$. In the calculated cross-sections the parameters of Woods-Saxon potential were chosen so that the inequality $\gamma_{N L x} \ll 2 \Delta E_{x}$ was true for the most of investigated cases.

Consequently, the electrons losing their energy $\omega_{v l \rightarrow N L}$ $\left(\omega_{0}-\Delta E_{x}<\omega_{v l \rightarrow N L}<\omega_{0}+\Delta E_{x}\right)$ during the excitation of a Coulomb resonance will be registered as electrons with the energies in the interval

$\varepsilon-\omega_{0}-\Delta E_{x} \leq \varepsilon^{\prime} \leq \varepsilon-\omega_{0}+\Delta E_{x}$. In this case the "regularized" cross-section $\sigma^{\text {reg }}\left(\omega, \theta^{\prime}\right)$ can be theoretically determined as

$$
\sigma^{r e g}\left(\omega, \theta^{\prime}\right)=\frac{1}{2 \Delta E_{x} \cdot \Omega_{x}} \int_{\omega-\Delta E_{x}}^{\omega+\Delta E_{x}} \iint_{\Omega_{x}} \sigma\left(\omega, \theta^{\prime}\right) \mathrm{d} \omega \mathrm{d} \Omega^{\prime} .
$$

It is necessary to note that the proposed above regularization practically does not change the functions in the regions of its smooth variation. In this connection, for example, we have: $\sigma^{(0) r e g}\left(\omega, \theta^{\prime}\right) \approx \sigma^{0}\left(\omega, \theta^{\prime}\right)$; $\sigma^{(d) r e g}\left(\omega, \theta^{\prime}\right) \approx \sigma^{d}\left(\omega, \theta^{\prime}\right)$ for : $\omega>60 \mathrm{MeV}$.

This "regularized" cross-section has resonant form in the area of Coulomb resonances and may have values comparable with the height of the quasi-elastic peak. Our further conclusions will be done in the assumption that we have only one isolated Coulomb resonance in the specified energy interval $2 \Delta E_{x}$. In the following we suppose that $2 \Delta E_{x}=1.0 \mathrm{MeV}$ for all experiments with various kinematics. Under these conditions we get predictive plots of experimental cross-sections $\sigma^{r e g}\left(\omega, \theta^{\prime}\right)$ of inelastic scattering of high-energy electrons on nuclei.

In the examples presented below we will investigate relatively large angles $\left(\theta_{0}^{\prime} \approx 1\right)$ of inelastic scattering of electrons. In this case the virtual photons emitted by relativistic electrons have relatively large $\left(\boldsymbol{q}^{2}-\omega^{2} \gg m^{2}\right)$ imaginary masses. This fact manifest itself, first of all, in reduction and disappearance of the dominant role of dipole transitions during the excitation of Coulomb resonances. Dipole, quadrupole, octopole transitions and transitions of even higher multi-polarity should be taken into account when one studies the spectra of inelastic largeangle scattering of high-energy electrons.

Very often the scattering of high-energy electrons is investigated with a carbon $\left({ }^{12} \mathrm{C}\right)$ target. This light nucleus is selected as standard for comparison of the efficiency of different kinematics in the identification of Coulomb resonances in the spectra of inelastic scattered electrons (Figure 1). One can see from the presented plot that, using the kinematics [15], one cannot observe the Cou-

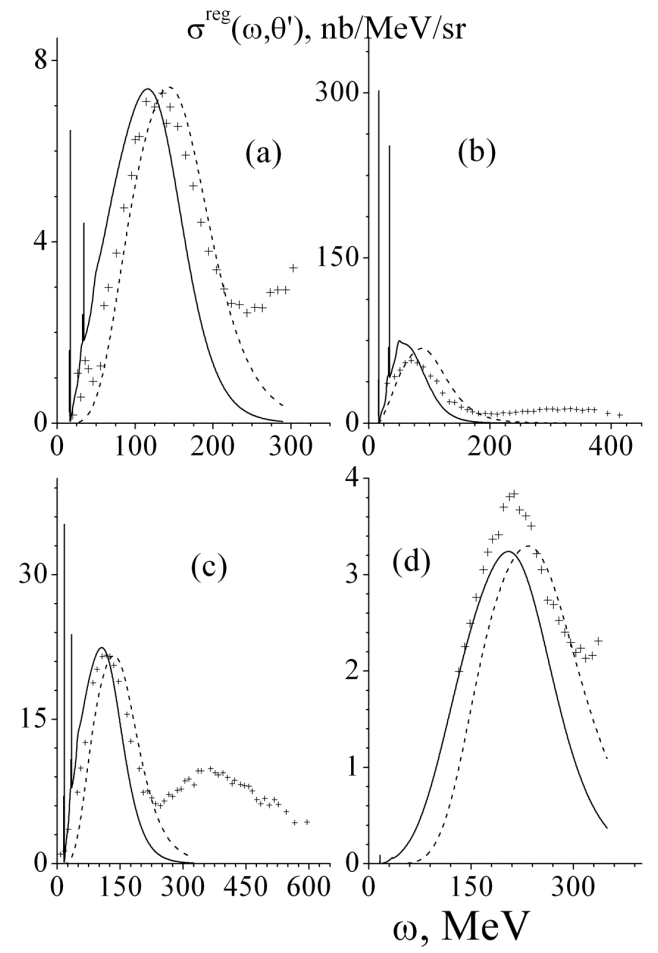

Figure 1. The inclusive and "regularized" cross-sections $\sigma^{\text {reg }}\left(\omega, \theta^{\prime}\right)$ of inelastic scattering of electrons on nuclei ${ }^{12} \mathrm{C}$ versus the initial energy of scattered electrons $\varepsilon$ and the angle of scattering $\theta^{\prime}$. The kinematics of scattering is selected as in fulfilled experiments [15]: $\varepsilon=500 \mathrm{MeV}, \theta^{\prime}=$ $60^{\circ}$ (a); $\varepsilon=537 \mathrm{MeV}, \theta^{\prime}=37^{\circ}$ (b); $\varepsilon=730 \mathrm{MeV}, \theta^{\prime}=37^{\circ}$ (c); $\varepsilon=779.5 \mathrm{MeV}, \theta^{\prime}=50.1^{\circ}$ (d). The parameters of Woods-Saxon potential are: $V_{0 p} / \mathrm{MeV}=61.412, V_{0 n} / \mathrm{MeV}=$ 55.684. Here and hereinafter the solid and the dashed curves were calculated, respectively, with allowance for the final-state interaction (regularized cross-sectins, $[(14),(36)]$ ), and in the plane-wave approximation (15).

lomb resonance with quantum numbers $N L=12$, which is theoretically predicted by the nuclear shell model, in the spectra of inelastically scattered electrons. It should be emphasized that this conclusion is wrong in the case of other kinematics presented on Figure 1. It is also pertinent to note that for the disclosure and identification of the resonances of the nucleus ${ }^{12} \mathrm{C}$ with the quantum numbers $N L x=12 p$ and $N L x=12 n$, it is necessary to carry out more thorough experimental measurements in the spectral region of interest, having essentially reduced the step of the argument $\Delta \omega$.

It is worth noting that the height of the regularized resonance peak on the graphs of $\sigma^{r e g}\left(\omega, \theta^{\prime}\right)$ corresponds to the height of the experimentally determined cross-section if the energy gap width of the recording device of scattering electrons is, as indicated above, $2 \Delta E_{x}=1 \mathrm{MeV}$. At the same time, the width of the resonance peak is equal to the theoretically calculated width of the investigated resonance. It is necessary to empha- 
size that the height of the Coulomb resonance essentially depends on the width of the energy gap of the recording device of electrons. If, for instance, the width of the energy gap of spectrometer is equal to $5 \mathrm{MeV}$, then the possibility of manifestation of Coulomb resonances in the spectra of inelastically scattered electrons strongly decreases.

One can conclude from Figure 1 that decreasing the initial energy $\varepsilon$ and the angle of scattering $\theta^{\prime}$ of electrons improves the conditions for observation of Coulomb resonances in the spectra of inelastically scattered electrons. Let us remind that the theoretically predicted heights of Coulomb and centrifugal resonances are comparable with the height of the quasi-elastic peak.

Note that the Woods-Saxon potential depth parameters ( $V_{0 p}$ and $V_{0 n}$ ) giving the highest accuracy were used, as a rule, in our calculations. The half-width $\gamma_{12}$ of a Coulomb resonance essentially increases (see Table 3) with a decrease of the depth of the Woods-Saxon potential. In this case the theoretically calculated Coulomb resonance $N L=12$ is widened so much that its half-width can considerably exceed the spectral energy gap of the spectrometer $2 \Delta E_{x}$. In this case the height of incipient Coulomb resonance decreases and this resonance can be identified directly in spectra of inelastically scattered electrons.

Figure 2 shows theoretically calculated regularized cross-sections $\sigma^{r e g}\left(\omega, \theta^{\prime}\right)$ of inelastical scattering of electrons and experimentally measured cross-sections $\sigma\left(\omega, \theta^{\prime}\right)$ of electro-disintegration on different nuclei $\left({ }^{12} \mathrm{C},{ }^{27} \mathrm{Al},{ }^{58} \mathrm{Ni},{ }^{198} \mathrm{Au}\right)$. The kinematics of the experiments is borrowed from [20].

Analysing the calculated $\sigma^{r e g}\left(\omega, \theta^{\prime}\right)$ (solid line) and $\sigma^{0}\left(\omega, \theta^{\prime}\right)$ (dashed line; plane wave approximation), we can observe some peculiarities of identification of Coulomb resonances in the spectra of inelastically scattered electrons in the framework of nuclear shell model. For example, one can conclude that the Coulomb resonances may be registered in all considered cases. But careful and painstaking measurements in the kinematics region interesting for us were not presented in [20]. Note also that in the case of heavy nuclei we have a large number of Coulomb, centrifugal, and incipient (with large halfwidth $\gamma_{v l \rightarrow N L} \approx 1 \div 2 \mathrm{MeV}$ ) [27] resonances. In this case the theoretical analysis of the full resonance picture of cross-section $\sigma^{r e g}\left(\omega, \theta^{\prime}\right)$ becomes more complex.

Finally at the Figure 3 are presented calculated crosssections $\sigma^{r e g}\left(\omega, \theta_{0}^{\prime}\right)$ for different nuclei ( $\mathrm{A} 1, \mathrm{Mg}, \mathrm{Ca}$, $\mathrm{Ni})$ and for the different kinematics: the initial energies of scattered electrons $\varepsilon=779.5 ; 500 ; 545 ; 500 \mathrm{MeV}$ and the angles of scattering $\theta^{\prime}=50.1^{\circ} ; 60^{\circ} ; 45^{\circ} ; 60^{\circ}$. The results of calculations presented on Figure 3, in general, do not contradict to the conclusions based on analysis of results presented at Figures 1 and 2. We can, however, state a fact that qualitative and quantitative comparisons of theoretical calculations and experimental data and
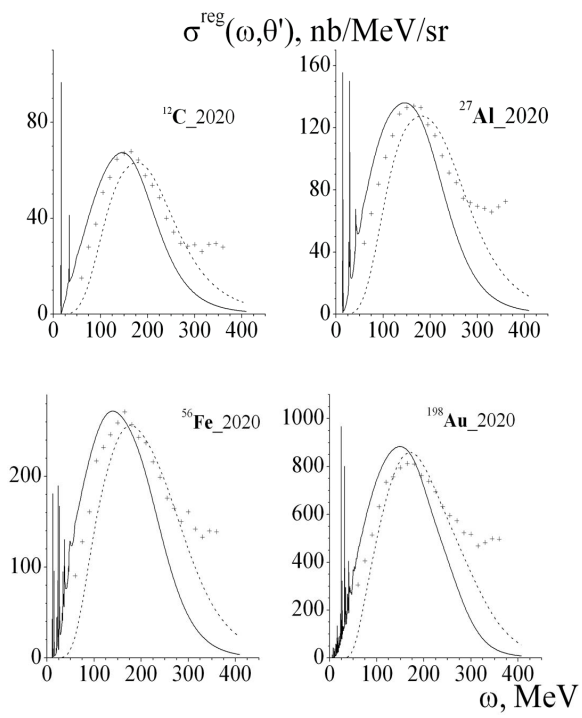

Figure 2. Regularized cross-section $\sigma^{r e g}\left(\omega, \theta^{\prime}\right)$ of inelastic scattering of electrons on the nuclei ${ }^{12} \mathrm{C},{ }^{27} \mathrm{Al},{ }^{56} \mathrm{Fe},{ }^{198} \mathrm{Au}$ versus the transmitted energy $\omega$. The initial energy of the scattered electrons is $\varepsilon=2020 \mathrm{MeV}$, the angle of scattering is $\theta^{\prime}=15^{\circ}$.

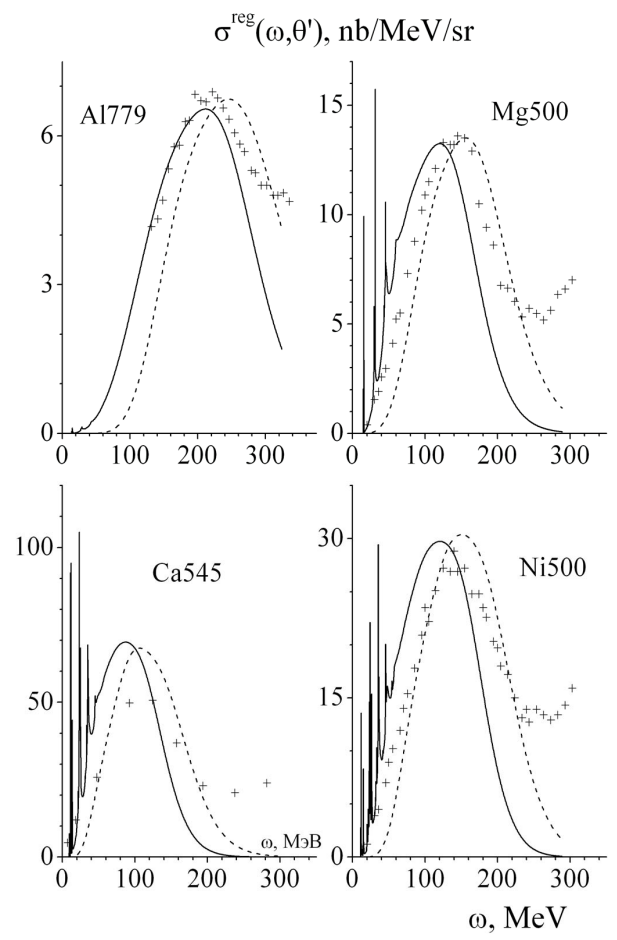

Figure 3. The cross-sections $\sigma^{\text {reg }}\left(\omega, \theta^{\prime}\right)$ of inelastic scattering of high-energy electrons on nuclei ${ }^{27} \mathrm{Al},{ }^{24} \mathrm{Mg},{ }^{40} \mathrm{Ca},{ }^{58} \mathrm{Ni}$ as a functions of the initial energy of scattered electrons $\varepsilon$ and angle of scattering $\theta^{\prime}$. Kinematics of scattering agrees with kinematics of experiments [9,15,22]: $\varepsilon=779.5 \mathrm{MeV}, \theta^{\prime}=$ $50.1^{\circ}(\mathrm{Al}), \varepsilon=500 \mathrm{MeV}, \theta^{\prime}=60^{\circ}(\mathrm{Mg}, \mathrm{Ni}) ; \varepsilon=545 \mathrm{MeV}, \varepsilon=$ 545 (Ca). The parameters of Woods-Saxon potential are: Al: $V_{0 p, 0 n}=63.5 ; 54.0(\mathrm{MeV})$; Mg: $V_{0 p, 0 n}=68.674 ; 60.1(\mathrm{MeV})$; Ca: $V_{0 p, 0 n}=53.9 ; 42.352(\mathrm{MeV}) ; \mathrm{Ni}: V_{0 p, 0 n}=62.85 ; 49.342$ (MeV). 
moreover the conclusions from such comparison are impossible at the moment. The reason is that experimental data and theoretical investigations are practically absent for the declared above range of transmitted energies.

\section{Quasielastic Peaks and Orthogonality of Wave Functions}

The interpretation of inelastic scattering of high-energy electrons and the reaction $A\left(e, e^{\prime} p\right)(A-1)$ considered here in the frames of nuclear shell model, despite of simplicity, remains self-consistent quantum-mechanical problem in the theory of nuclei. The main reason and decisive argument for this statement is such well-known property as the mutual orthogonality of the wave functions [7] of discrete and continuous, including the quasidiscrete, spectra. Notice that the term "orthogonal functions" in aspect of interpretation of processes of electro-disintegration of nuclei by the high-energy electrons was unambiguously used in [7]. In addition to stated in [7] one may note that it is very difficult to imagine quantum-mechanical theory of quantum transitions as well as based on it theory of inelastic scattering without complete orthogonal basis of wave functions of investigated quantum-mechanical system. We emphasize also that the conclusions below are based only on the postulates of the nuclear shell model and may be used for the investigation of different aspects of this model.

In order to illustrate the role of orthogonality of wave functions in the theory of electro-disintegration of nuclei, let us consider the process of small-angle $\left(\theta^{\prime}=15^{\circ}\right)$ and very small-angle scattering (quasi-real photons phenomenon: $\left.\theta^{\prime} \ll \frac{m \omega}{\varepsilon \varepsilon^{\prime}} \ll 1\right)$. Let us now calculate the crosssections $\left.\sigma\left(\omega, \theta^{\prime}\right)\right|_{\theta^{\prime} \ll 1}$ of inelastic scattering of highenergy electrons on nucleus ${ }^{12} \mathrm{C}$ for two values of initial energy of electrons, $\varepsilon=500 \mathrm{MeV}$ and $\varepsilon=2020 \mathrm{MeV}$.

First, let us remind that the dominant position in the expression for the exclusive cross section belongs to two functions of kinematical variables, $P\left(\boldsymbol{k}, \boldsymbol{k}^{\prime}\right)$ (Equation (13))) and $G_{x v l}(\boldsymbol{q}, \boldsymbol{K})$ (Equation (14))). It is more than appropriate mention here that the latter one is determined by overlap integral:

$$
I_{x v l m}\left(\boldsymbol{q}, \boldsymbol{K}_{x}\right)=\iiint_{-\infty}^{(-)^{*}}(\boldsymbol{r})(\exp (i \boldsymbol{q} \boldsymbol{r})) \phi_{x v l m}(\boldsymbol{r}) \mathrm{d}^{3} \boldsymbol{r} .
$$

Taking into consideration the formula cited above, in an early stage of our analysis let us suppose that $|\boldsymbol{q}|=0$. Therefore, $\exp (i q r)=1$. Then, due to the orthogonality condition of the wave functions of discrete and continuous spectra, $\left.I_{x v l m}\left(\boldsymbol{q}, \boldsymbol{K}_{x}\right)\right|_{q=0}=0$. It should be noted that this case $(|\boldsymbol{q}|=0)$ is unacceptable. If $|\boldsymbol{q}|=0$ then inelastic (and elastic) scattering is impossible.

Second, let us now suppose that $|\boldsymbol{q}|=R^{-1}$. In this case $\exp (i q R) \approx 1 \quad(R$ is the radius of the nucleus $)$ and $\left|R^{-3 / 2} I_{x v l m}\left(\boldsymbol{q}, \boldsymbol{K}_{x}\right)\right| \ll 1$. This latter case $(|\boldsymbol{q}| \neq 0)$ is possible. In this case the losses of momentum and energy of the scattered electron must be minimal. If the value of transmitted energy $\omega$ is fixed then the minimal value of transmitted momentum takes place at the minimum angle of scattering ( $\theta^{\prime}=0$, quasi-real photons). As this is the case, $|\boldsymbol{q}| \approx \omega$. And, as a rule, the inequality $\left(\frac{\boldsymbol{q}^{2}-\omega^{2}}{\omega^{2}}\right) \ll 1$ takes place as well $[25,27]$.

The minimal value of the energy $\omega$ necessary for the excitation of Coulomb resonances is practically defined by the minimal excitation energy of the lowest Coulomb resonance, $E_{p N L}+\left|\varepsilon_{x v l}\right|$, where $\varepsilon_{x v l}$ is the proton energy in the highest filled nuclear shell. Hence, the inequality $|\boldsymbol{q}| \ll R^{-1}$ may be satisfied with reasonable accuracy. The numerical estimates for the investigated nuclei lead to the following result: $|\boldsymbol{q}|_{\min } \approx 0.05 \mathrm{Fm}^{-1}$. So, it is natural that the inequality $|\boldsymbol{q}| \ll R^{-1}$, in turn, leads to the inequality $\left|R^{-3 / 2} I_{x v l m}\left(\boldsymbol{q}, \boldsymbol{K}_{x}\right)\right| \ll 1$, which has already been mentioned.

Thus, restricting ourselves only with the analysis of phenomenon of orthogonality of wave functions of discrete and continuous spectra, we may predict decrease of the cross-section of inelastic scattering of high-energy electrons. This decrease takes place at small transmitted energies $\omega$ and small scattering angles $\left(\theta^{\prime} \ll 1\right)$ of electrons. Note that this phenomenon is caused by decreasing of distorted momentum distribution $G_{x v l}^{(d)}(\boldsymbol{q}, \boldsymbol{K})$. In the case of plane-wave approximation this statement is wrong because the plane wave $\exp (i \boldsymbol{K r})$ is essentially nonorthogonal to the wave function of bounded state of the proton in the considered kinematical region.

Let us recall that the expression for the cross-section $\sigma\left(\omega, \theta^{\prime}\right)$ has dimensionless factor $P\left(\boldsymbol{k}, \boldsymbol{k}^{\prime}\right)$ that, as it well known [25,27], is large in the mentioned above region. Just the function $P\left(\boldsymbol{k}, \boldsymbol{k}^{\prime}\right)$ due to nonorthogonality of $\exp (i \boldsymbol{K} \boldsymbol{r})$ and $\varphi_{x v l m}(\boldsymbol{r})$ will lead to unacceptably huge values of cross-section in plane-wave approximation. And just this factor allows us to draw an unambiguous conclusion concerning the significance of taking into account of orthogonality of wave functions of discrete and continuous spectra.

The phenomenon of orthogonality of wave functions in our calculations is represented on Figure 4, where plotted the cross-sections $\sigma\left(\omega, \theta^{\prime}\right)$ of inelastic scattering of electrons on nucleus ${ }^{12} \mathrm{C}$. The parameters of Woods-Saxon potential were chosen in such way that the Coulomb $(N L=20,12)$ and centrifugal $(N L=12)$ resonances have relatively large visually observed half-width.

The cross-sections calculated in the plane-wave approximation significantly exceed $\sigma^{(d)}\left(\omega, \theta^{\prime}\right)$ calculated with taking into account of interaction of the knocked- 


$$
\sigma^{(1)}(\omega, \theta) \cdot \mathrm{mkb} / \mathrm{MeV} / \mathrm{sr}
$$
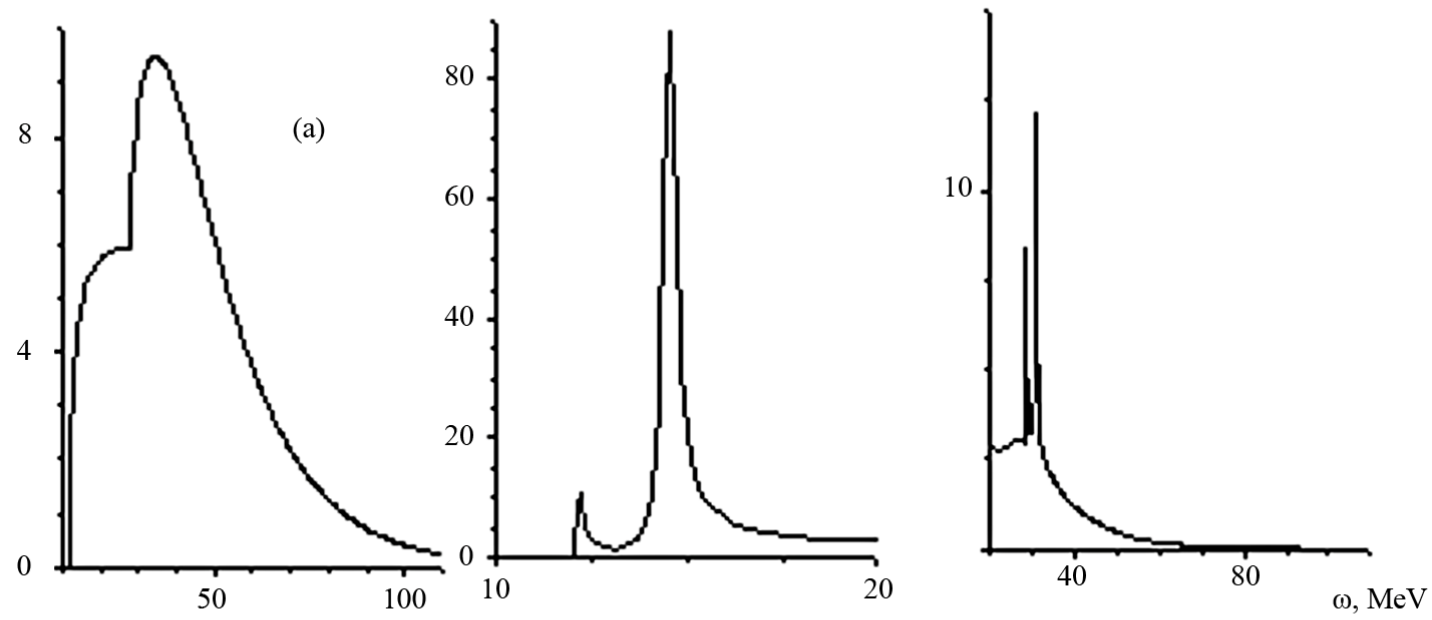

$\sigma^{(2)}(\omega, \theta) \cdot \mathrm{Gb} / \mathrm{MeV} / \mathrm{sr}$
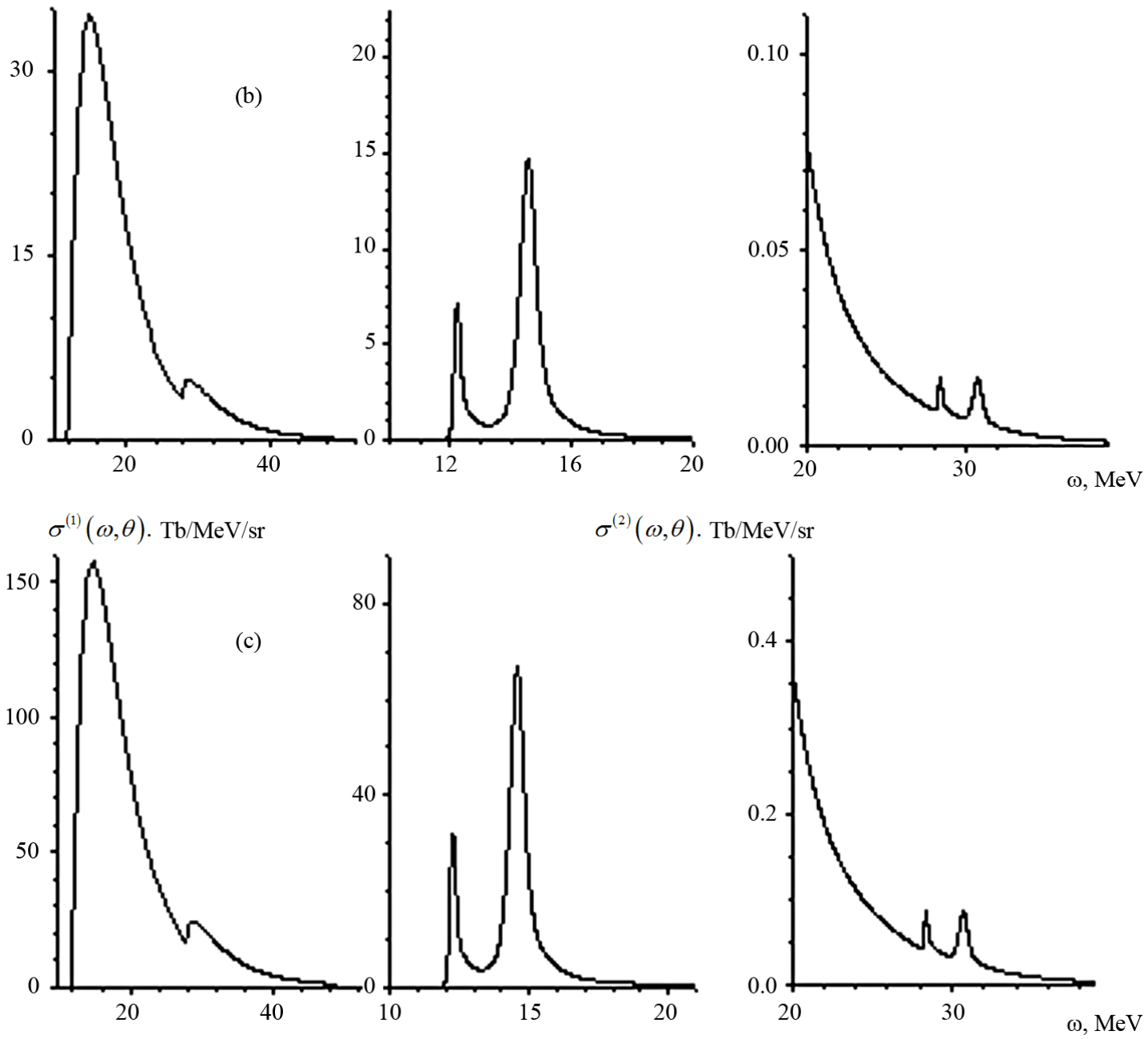

Figure 4. The cross-sections $\sigma\left(\omega, \theta^{\prime}\right)$ of inelastic scattering of electrons on the nucleus ${ }^{12} \mathrm{C}$ versus the transmitted energy $\omega$. The parameters of the Woods-Saxon potential: $V_{0 p}=53.7 \mathrm{MeV}, V_{0 n}=51.0 \mathrm{MeV}$; the initial energies of the electrons: $\varepsilon=500$ $\mathrm{MeV}(\mathrm{a}, \mathrm{b}) ; \varepsilon=2020 \mathrm{MeV}(\mathrm{c}) ;$ the angles of scattering are: $\theta^{\prime}=15^{\circ}(\mathrm{a}) ; \theta^{\prime}=\left[3 \times 10^{-9}\right] \ll \frac{m \omega}{\varepsilon \varepsilon^{\prime}} \quad(\mathrm{b}, \mathrm{c})$. 
out nucleon in the final state, see Figure 4. Note that after some doubts and speculations the reader cannot disturb himself and fear of unusual units $(\mathrm{Gb}, \mathrm{Tb})$ that appear at the Figure 4. These units will be usual and natural units in the near future.

The plane-wave cross-sections have huge, nonphysical magnitudes in the region of the quasi-elastic peak. This fact is a convincing proof of the necessity of taking into account the interaction of the knocked-out nucleon with the residual nucleus in the final state.

\section{Coulomb Resonances, Quasi-Real Photons and $\chi$-Experiments}

In this section we will attempt to interpret some new aspects of experiments on investigation of exclusive crosssections $[10,15,23]$ basing on the results above. These new cross-sections

$$
\chi\left(E_{\text {miss }}\right)=\frac{\mathrm{d}^{6} \sigma}{\mathrm{d} \varepsilon^{\prime} \mathrm{d} E \mathrm{~d} \Omega^{\prime} \mathrm{d} \Omega}
$$

depend on the energy $E_{\text {miss }}$ lost in processes of scattering and re-scattering of the inelastically scattered electron and the knocked-out high-energy proton in the initial and final states. In such experiments one fixes the cases of simultaneous registration of scattered electron with the energy $\varepsilon^{\prime}$ and knocked-out proton with the energy $E$ $(E \approx 100 \mathrm{MeV})$. As this takes place, we can easily determine experimentally the lost energy $E_{\text {miss }}$ : $\varepsilon=\varepsilon^{\prime}+E+E_{\text {miss }}$. The plots of $\chi\left(E_{\text {miss }}\right)$ presented in $[10,15,23]$ have weak resonance nature. For example, in the case of the nucleus ${ }^{12} \mathrm{C}$ the function $\chi\left(E_{\text {miss }}\right)$ have a sharp maximum at $E_{\text {miss }} \approx 17 \mathrm{MeV}$ and a smooth maxi- mum at $E_{\text {miss }} \approx 40 \mathrm{MeV}$. At the same time, $\chi\left(E_{\text {miss }}\right) \approx 0 \forall\left[E_{\text {miss }}<E_{\text {miss }}^{0} \approx 17 \mathrm{MeV}\right]$.

Within the framework of nuclear shell model the most convenient interpretation of the minimal lost energy is $E_{\text {miss }}=\varepsilon_{11}^{h}, \varepsilon_{11}^{h}>0$ is the binding energy at the upper filled shell of nucleus ${ }^{12} \mathrm{C}$.

If $E_{\text {miss }}>\varepsilon_{v l}^{h}$, then additional losses of energy $\Delta E_{\text {miss }}=E_{\text {miss }}-\varepsilon_{v l}^{h}$ may be interpreted as the result of one or several phenomena listed below. These phenomena are: strong interaction of the knocked-out proton with the residual nucleus $A-1$ in the final state $[15,16]$, which is accompanied by excitation of this nucleus; the knocking-out of protons out of deeper filled nuclear shells; losses of energy of the scattered electron in initial state; accidental coincidences that are caused by the existence of intensive background of protons, the source of which is [25] the scattering of high-energy electrons caused by quasi-real photons; the dispersion of the initial energies of scattered electrons in the incident beams; etc.

At the initial stage of investigations it is reasonable to look for the cause of additional energy losses
$\Delta E_{\text {miss }}=E_{\text {miss }}-\varepsilon_{v l}^{h}$ in strong interaction $[15,16]$ of the knocked-out proton with the residual nucleus in the finite state. Note that in this case we suppose that the probability of inelastic re-scattering of the weakly interacting high-energy electron on nucleons of the residual nucleus or another nucleus of the target is negligible.

Let us suppose that one of the protons of an upper shell of the nucleus receives the energy $\omega=\varepsilon-\varepsilon^{\prime}$ after an act of collision with a high-energy electron. This proton with the energy $E_{p}=\omega-\varepsilon_{v l}^{h}$ can participate in the processes of inelastic scattering on residual $(A-1)$ nucleus too. In other words, the knocking-out strongly interacting proton spent the part of acquired energy in the processes of inelastic re-scattering on that $(A-1)$ or other $A$ atomic nucleus. The knocking-out strongly interacting proton may spent, for instance, the part of its energy for the excitation of residual nucleus or increase the number of knocking-out nucleons in the processes of collisions with other nucleons, etc. Let us suppose that the processes of re-scattering of knocking-out protons in the finite state play an important role in our investigation. Then it is evident that these processes can essentially complicate the interpretation of processes of electrodisintegration of atomic nuclei in coincidence experiments. We intend to propose another alternative interpretation of origin of additional losses of energy in $\chi$ experiments $[10,15,23]$. Let us proceed from the anomalously large values of the scattering cross-sections caused by quasi-real photons ( $\theta^{\prime}=0$, , $0-0 "$ - scattering).

We noticed above that in the analyzed experiments the coincidence of two particles was recorded. These particles are: the inelastically scattered electron $k^{\prime}$ and knocking-out (it is very desirable that just by this electron) proton $K$. Using simple empirical conception one can state that knocking-out of proton from some nucleus ${ }^{12} \mathrm{C}$ unambiguously testify that the scattered electron reside inside (or in the immediate vicinity) of this nucleus. It is not difficult estimate the length $\lambda$ of mean free path of relativistic electron inside of this nucleus knowing the cross-section of excitation of Coulomb resonance in nucleus ${ }^{12} \mathrm{C}$ by electrons with the energy $\varepsilon=779 \mathrm{MeV}$. As a result of the estimate we can assert that such high-energy electron in the process of collision with nucleus ${ }^{12} \mathrm{C}$ before or after of knockout of proton may excite the Coulomb resonance $N L=12$ in this (or another) nucleus with large probability.

Previously developed theory $[12,25,27]$ of Coulomb resonances and presented above estimates based on the calculated data of cross-sections of excitation of Coulomb resonances stimulate us to state such hypothesis in contrary to primordially widespread opinion: the lost energy $E_{\text {miss }}$ in experiments $[10,15,23]$ is the energy that is used for the excitation of discrete, quasi-discrete and continuous spectra of investigated nucleus but namely 
by the high-energy electrons. Note that in this case the main role belongs to quasi-real photons $\left(\theta^{\prime}=0\right.$, , $0-0$ " scattering).

In connection with the hypothesis presented above we should say the following. Let us suppose for a while that the results presented in Tables 1-5 are unknown. In this case the hypothesis stated above is, at least, a striking and very glaring example of absurdity, irresponsibility, and nonsense.

The origin of the hypothesis stated above is in results of works $[12,25,27]$ and in above-presented calculations of the cross-sections of the excitation of Coulomb resonances. In other words, the hypothesis mentioned above is undoubtedly based on the processes that are introduced to our theory by the Coulomb resonances and quasi-real photons. It is worth noting that the experiment can verify or refute this hypothesis, but in both cases we will receive equally important information.

In order to confirm or to refute the hypothesis presented above, we suggest the modification of experiments $[10,15,23]$ already mentioned above. Consider, for instance, the experiment [15] where the inclusive crosssection $\chi\left(E_{\text {miss }}\right)$ of electro-disintegration of nucleus ${ }^{12} \mathrm{C}$ by high-energy ( $\varepsilon=779.5 \mathrm{MeV}$ ) electrons is investigated. The measurement of $\chi\left(E_{\text {miss }}\right)$ is merely the first stage of slightly extended experiment. The final stage of suggested experiment is practically the same. The physicist must measure the cross-section $\chi\left(E_{\text {miss }}\right)$ in slightly changed configuration.

The important but practically negligible and easily implemented modification of presented configuration is in additional auxiliary carbonic target installed at the very entry of spectrometer of scattered electrons. For preliminary estimate of thickness of supposed auxiliary carbonic target we may use the following speculations. Let we have $1 \mathrm{~cm}^{2}$ of target of the etalon density $(D=100$ $\left.\mathrm{mg} / \mathrm{cm}^{2}\right)$. This carbonic target contains $100 \times 10^{-3} /(1.66$ $\left.\times 10^{-24}\right) \approx 6 \times 10^{22}$ nucleons. A half of these nucleons are protons. The Table 3 contains calculated cross-sections of excitation of Coulomb resonances of the nucleus ${ }^{12} \mathrm{C}$. According to this Table, each of four protons of the $1 p$-shell of this nucleus is an impermeable shield for the high-energy $(\varepsilon \approx 700 \mathrm{MeV})$ electrons that excite the Coulomb resonance $[N L=21]$ of the investigated nucleus. The area of this shield is $20 b=20 \times 10^{-24} \mathrm{~cm}^{2}$. Thus the total impermeable shield of $1 \mathrm{~cm}^{2}$ of our etalon target will be $20 \times 10^{-24} \times 2 \times 10^{22} \mathrm{~cm}^{2} \approx 0.4 \mathrm{~cm}^{2}$. The obtained result testifies that about $40 \%$ of electrons moving through the target and having the direction of movement unchanged will have the energy less by 17 $\mathrm{MeV}$ than in the incident beam. Thus, according to the calculations, the density $D$ of our additional target must be $D \approx 240-260 \mathrm{mg} / \mathrm{cm}^{2}$.

If the hypothesis about the origin of energy losses
$E_{\text {miss }}$ in our measurements is valid then in the modified configuration we must obtain practically the same picture of the cross-section $\chi\left(E_{\text {miss }}\right)$. The only difference between the two cross-sections is that the latter will be shifted to the right along the axis $E_{\text {miss }}$ by the value $\Delta E_{\text {miss }} \approx 17 \mathrm{MeV}$. Note that the simplest version of modification of experiment [15], which was proposed above, may be diversified and complicated, for instance, by varying the thickness of the additional target on the entry slit of the electron/proton spectrometer or by choosing another sort of nuclei for the additional target. At the moment, a more detailed analysis of such variations is, at least, premature.

Finally, we can conclude that the investigation of inelastic scattering of high-energy electrons and, particularly, the experimental study of Coulomb resonances and quasireal photons is of general theoretical importance, especially, for quantum nonrelativistic mechanics.

\section{Conclusions}

The main results of present paper can be summarized and briefly stated in the following way:

- Coulomb resonances are the direct theoretical prolongation of the nuclear shell structure to the continuous spectrum region. In the framework of the oneparticle(!) theory of Coulomb resonances one can readily explain many features of such well-known phenomenon as dipole (quadrupol, octopol,...) giant resonance. It is a real possibility to interpret the phenomenon of dipole giant resonance as real experimental confirmation of existence of the Coulomb resonances in the atomic nuclei. The brief theory of Coulomb resonances and calculated quasi-discrete spectra of some atomic nuclei are presented in Sections 2 and 3.

- The regularized cross sections calculated in this paper convince of the theoretical possibility of direct manifestation of Coulomb resonances in the spectra of inelastically scattered high energy electrons. The presented calculations allow us to suggest the best kinematic conditions for observation of such manifestation:

- initial energies of high energy electrons, $\varepsilon \approx 300-500$ $\mathrm{MeV}$;

- electron scattering angles, $\theta^{\prime} \approx 10-25$ degrees.

The necessary condition for such manifestation is substantially painstaking measurement of cross sections of inelastic scattering of high energy electrons with high resolution in the region of transmitted energy, $5 \mathrm{MeV} \leq$ $\omega \leq 60 \mathrm{MeV}$ (Section 3).

- To emphasize the significance of the wave functions orthogonality (both initial and final states of nuclei) one may investigate the scattering of high energy 
electrons at very small angles $\theta^{\prime} \ll 1$ (Section 4).

- The main kinematic peculiarity of virtual quasi-real photons is the approximate equality of their quasimomentum $q$ and energy $\omega: q^{2} \approx \omega^{2}$. This approximate equality determines the effective capability of quasi-real photons for knocking-out of protons of high nuclear shells into quasi-discrete states of nuclei. In other words, quasi-real photons excite Coulomb resonances of atomic nuclei with high efficiency. The cross sections of such exciting processes can result in huge, almost tremendous values that may be equal to hundreds and thousands of barns depending on the initial value of energy $\varepsilon$ of scattering electrons. Such processes can be discovered in experiments proposed in this paper (Section 5).

\section{Acknowledgements}

Authors wish to thank Dr. V. V. Lutsenko and Dr. Yu. V. Yakovenko for their sincere efforts in improvement of the style of the language of submitted text of this article.

\section{REFERENCES}

[1] A. G. Sitenko and V. N. Gur'ev, "Inelastic Scattering of High Energy Electrons on Nuclei," Soviet Physics - JETP, Vol. 12, No. 8, 1960, pp. 1228-1234.

[2] K. W. McVoy and L. Van Hove, "Inelastic ElectronNucleus Scattering and Nucleon-Nucleon Correlations," Physical Review, Vol. 125, No. 3, 1962, pp. 1034-1043. doi:10.1103/PhysRev.125.1034

[3] W. Czyz, "Inelastic Electron Scattering from Nuclei and Single-Particle Excitations," Physical Review, Vol. 131, No. 5, 1963, pp. 2141-2148. doi:10.1103/PhysRev.131.2141

[4] V. Devanathan, "Quasi-Free Scattering of Electrons and Nuclear Shell Structure," Annals of Physics, Vol. 43, No. 1, 1967, pp.74-90. doi:10.1016/0003-4916(67)90292-8

[5] V. K. Tartakovskii and A. A. Pasichnyi, "Inelastic Scattering of High-Speed Electrons by Nuclei with Nucleon Knockout," Ukrainian Journal of Physics, Vol. 13, No. 8, 1968, pp. 1361-1368.

[6] A. A. Pasichnyi and V. K. Tartakovskii, "Coupled One-Particle States of Nucleons in a Spherical Potential," Ukrainian Journal of Physics, Vol. 13, No. 12, 1968, pp. 2013-2019.

[7] A. G. Sitenko, A. A. Pasichnyi and V. K. Tartakovskii, "On Electrodisintegration of Light Nuclei," Soviet Journal of Nuclear Physics, Vol. 12, 1970, pp. 663-672.

[8] A. A. Pasichnyi, "Study of Nuclear Shell Structure in Inelastic High Energy Electron Scattering," Ukrainian Journal of Physics, Vol. 17, No. 7, 1972, pp. 1130-1138.

[9] R. R. Whitney et al., "Quasielastic Electron Scattering," Physical Review C, Vol. 9, No. 6, 1974, pp. 2230-2235. doi:10.1103/PhysRevC.9.2230

[10] J. Moygey, et al., "Quasi-Free (e, e'p) Scattering on ${ }^{12} \mathrm{C}$,
${ }^{28} \mathrm{Si},{ }^{40} \mathrm{Ca}$ and ${ }^{58} \mathrm{Ni}, "$ Nuclear Physics A, Vol. 262, No. 3, 1976, pp. 461-492. doi:10.1016/0375-9474(76)90510-8

[11] J. S. O'Connell, et al., "Electromagnetic Excitation of the Delta Resonance in Nuclei," Physical Review C, Vol. 35, No. 3, 1987, pp. 1063-1071. doi:10.1103/PhysRevC.35.1063

[12] A. A. Pasichnyi, "On Dynamics of Electrodisintegration of Nuclei at Low Energies," Soviet Journal of Nuclear Physics, Vol. 54, No. 12, 1991, pp. 944-955.

[13] A. A. Pasichnyi, "Coulomb Resonances and Nuclear Disintegration," Ukrainian Journal of Physics, Vol. 37, No. 4, 1992, pp. 487-497.

[14] M. Zhongyu and F. Dachun, "Quasielastic Electron Scattering in Quasiparticle Approach," Physical Review C, Vol. 45, No. 2, 1992, pp. 811-818. doi:10.1103/PhysRevC.45.811

[15] G. Garino, et al., "Proton Propagation in Nuclei Studied in the (e,e'p) Reaction," Physical Review C, Vol. 45, No. 2, 1992, pp. 780-790. doi:10.1103/PhysRevC.45.780

[16] V. R. Pandharipande and S. C. Pieper, "Nuclear Transparency to Intermediate-Energy Nucleons from (e,e'p) Reactions," Physical Review C, Vol. 45, No. 2, 1992, pp. 791-798. doi:10.1103/PhysRevC.45.791

[17] Y. H. Jin, D. S. Onley and L. E. Wright, "Electron Coulomb Effects in Quasielastic (e,e'p) Reactions," Physical Review C, Vol. 45, No. 3, 1992, pp. 1311-1320. doi:10.1103/PhysRevC.45.1311

[18] A. A. Pasichnyi, "Dynamics of Electrodisintegration of Nuclei at Low Energies," Proceedings of the 8th Seminar on Electromagnetic Nuclear Interactions at Low and Intermediate Energies, Nauka, January 1992, pp. 296- 299.

[19] A. A. Pasichnyi, "Quasi-Discrete Spectra of Atomic Nuclei," Ukrainian Journal of Physics, Vol. 38, No. 11, 1993, pp. 1619-1630.

[20] D. B. Day, et al., "Inclusive Electron-Nucleus Scattering at High Momentum Transfer," Physical Review C, Vol. 48, No. 4, 1993, 1849-1863. doi:10.1103/PhysRevC.48.1849

[21] A. I. Akhiezer, A. G. Sitenko and V. K. Tartakovskii, "Nuclear Electrodynamics," Springer, Berlin, 1994.

[22] C. F. Williamson et al., "Quasielastic Electron Scattering from ${ }^{40} \mathrm{Ca}$," Physical Review C, Vol. 56, No. 6, 1997, pp. 3152-3172. doi:10.1103/PhysRevC.56.3152

[23] D. Dutta, et al., "Separated Spectral Functions for the Quasifree ${ }^{12} \mathrm{C}(\mathrm{e}, \mathrm{e}$ 'p) Reaction," Physical Review C, Vol. 61, No. 6, 2000, Article ID: 061602.

[24] A. Meucci, C. Giusti and F. D. Pacati, "Relativistic Corrections in (e,e'p) Knockout Reactions," Physical Review C, Vol. 64, No. 1, 2001, Article ID: 014604. doi:10.1103/PhysRevC.64.014604

[25] A. A. Pasichnyi and O. A. Prigodyuk, "On the Electrodisintegration of Nuclei," Physics of Atomic Nuclei, Vol. 68, No. 12, 2005, pp. 1988-2008.

[26] O. Benhar, D. Day and I. Sick, "Inclusive Quasielastic Electron-Nucleus Scattering," Reviews of Modern Physics, Vol. 80, No. 1, 2008, pp. 189-224. 
doi:10.1103/RevModPhys.80.189

[27] A. A. Pasichnyi, "The Coulomb Resonances and Dynamics of Electrodisintegration of Nuclei by High-Energy Electrons," Physics of Particles and Nuclei, Vol. 41, No. 1, 2010, pp. 108-148. doi:10.1134/S1063779610010065

[28] J. J. Sakurai, “Advanced Quantum Mechanics,” Addison Wesley Publishing Company, Reading, 1967.

[29] V. V. Balashov, "Quantum Theory of Collisions," Moskow University, Moskow, 1985, in Russian.

[30] V. B. Berestetskii, E. M. Lifshitz and L. P. Pitaevskii, "Course of Theoretical Physics, Quantum Electro-dynamics," Vol. 4, Pergamon, Oxford, 1982.

[31] A. S. Davydov, "Theory of the Atomic Nucleus," Nauka, Moscow, 1958 (in Russian).
[32] D. A. Varshalovich, A. N. Moskalev and V. K. Hersonskiy, "Quantum Theory of Angular Momentum," World Scientific, Singapore, 1988.

[33] S. E. Koonin, "Computational Physics," Addison Wesley Publishing Company, London, 1986.

[34] H. F. Lutz and M. D. Karvelis, "Numerical Calculation of Coulomb Wave Functions for Repulsive Coulomb Fields," Nuclear Physics, Vol. 43, No. 1, 1963, pp. 31-44. doi:10.1016/0029-5582(63)90325-0

[35] M. Abramowitz and I. A. Stegun, "Handbook of Mathematical Functions," National Bureau of Standarts: Applied Mathematics Series 55, 1964. 


\section{Appendix}

\section{A. Calculation of the Electro-Disintegration Cross Sections and Testing of Numerical Programs}

The accurate calculation of the cross sections of inelastic electron scattering is connected with the accurate calculation of the overlap integrals (18), which are the most important components of Equation (8). That problem will be investigated in detail below. Note that the integrand in Equation (18) is the product of the exponent $\exp (i \boldsymbol{q} r)$, the bound-state wave function $\varphi_{x v l m}(\boldsymbol{r})$ and the wave function of continuous spectrum $\psi_{K}^{ \pm}(\boldsymbol{r})$. For the Woods-Saxon potential, the wave function of the bound state $\varphi_{x v l m}(\boldsymbol{r})$ is well known [6]. This function can be written as

$$
\begin{aligned}
& \varphi_{x v l m}(\boldsymbol{r})=R_{x v l}(r) Y_{l m}\left(\boldsymbol{n}_{r}\right) \equiv A_{x v l} \frac{Z_{x v l}(r)}{r} Y_{l m}\left(\boldsymbol{n}_{r}\right) ; \\
& A_{x v l}^{2} \int_{0}^{\infty} Z_{x v l}^{2}(r) \mathrm{d} r=1 .
\end{aligned}
$$

The form and the content of the expressions presented above for $\varphi_{x v l m}(\boldsymbol{r})$ and $\psi_{K}^{(-)}(\boldsymbol{r})$ together with the requirement that the cross-section calculations are of high precision predetermine the choice of the integration method in Equation (18).

To calculate the overlap integral (18), we use the wellknown plane-wave expansion [31,32]:

$$
\mathrm{e}^{i \boldsymbol{q} \boldsymbol{r}}=4 \pi \sum_{l_{2}=0}^{l_{2}=\infty} \sum_{m_{2}=-l_{2}}^{m_{2}=l_{2}} i_{l_{2}}^{l_{2}} j_{l_{2}}(q r) Y_{l_{2} m_{2}}^{*}\left(\boldsymbol{n}_{r}\right) Y_{l_{2} m_{2}}\left(\boldsymbol{n}_{q}\right), \boldsymbol{n}_{q}=\frac{\boldsymbol{q}}{|\boldsymbol{q}|}
$$

After putting Equations (22), (A1), and (A2), into Equation (18), we present $I_{x v l m}\left(\boldsymbol{q}, \boldsymbol{K}_{x}\right)$. in the following form:

$$
\begin{aligned}
& I_{x v l m}\left(\boldsymbol{q}, \boldsymbol{K}_{x}\right)=(4 \pi)^{2} \sum_{l_{1}=0}^{l_{1}=\infty} \sum_{m_{1}=-l_{1}} \sum_{l_{2}=0} \sum_{m_{2}=-l_{2}} i_{l_{2}}=\infty \\
& l_{m_{2}}=l_{2} \\
& \times \sqrt{\frac{\left(2 l_{2}+1\right)(2 l+1)}{4 \pi\left(2 l_{1}+1\right)}}\left(l_{2} 0 l 0 \mid l_{1} 0\right) \\
& \times\left(l_{2} m_{2} l m \mid l_{1} m_{1}\right) A_{l_{1} l_{2} v l}(q, K) Y_{m_{1}}^{l_{1}}\left(\boldsymbol{n}_{K}\right) Y_{m_{2}}^{l_{2}}\left(\boldsymbol{n}_{\boldsymbol{q}}\right),
\end{aligned}
$$

where

$$
A_{l_{1} l_{2} v l}(q, K)=\int_{0}^{\infty} R_{K l_{1}}(r) j_{l_{2}}(q r) R_{x v l}(r) r^{2} \mathrm{~d} r
$$

and $\left(l_{2} m_{2} l m \mid l_{1} m_{1}\right)$ are the Clebsh-Gordon [31,32] coefficients.

The overlap integral is presented in Equation (A3) in vector form, that is to say, Equation (A3) is valid in any arbitrarily chosen reference frame. We can take advan- tage of this freedom of choice and simplify Equation (A3). With this purpose, we recall that

$$
\left.Y_{m}^{l}(\boldsymbol{n})\right|_{\boldsymbol{n}=\boldsymbol{e}_{z}}=\delta_{m 0} \sqrt{\frac{2 l+1}{4 \pi}}
$$

If we superpose vector $\boldsymbol{e}_{z}$ with vector $\boldsymbol{q}$ or $\boldsymbol{K}$ then, according to Equation (A5), the summation in Equation (A3) is essentially simplified.

We restrict ourselves to the case of $\boldsymbol{e}_{z} \| \boldsymbol{q}$ and make use of well-known [32] properties of the Clebsh-Gordon coefficients. After that, the overlap integral takes the form

$$
\begin{aligned}
& I_{x v l m}\left(\boldsymbol{q}, \boldsymbol{K}_{x}\right)=\left(4 \pi i^{-l} \sqrt{2 l+1}\right) \sum_{l_{1}=0}^{l_{1}=\infty} \sum_{j=0}^{j=l}(-1)^{j} \sqrt{\frac{1}{2 l_{1}+1}} \\
& \times\left(\left(l_{1}-l+2 j\right) 0 l 0 \mid l_{1} 0\right) \times\left(\left(l_{1}-l+2 j\right) 0 l m \mid l_{1} m\right) \\
& \times A_{l_{1} l_{2} v l}(q, K) Y_{m}^{l_{1}}\left(\boldsymbol{n}_{\boldsymbol{K}}\right) \forall:\left(l_{2}=l_{1}-l+2 j\right) \text { and }\left(\boldsymbol{e}_{z} \| \boldsymbol{q}\right)
\end{aligned}
$$

Notwithstanding the achieved facilitation, the overlap integral (A6) remains a very complicated expression in the form of a twofold series, the convergence of which depends essentially on the kinematic parameters. It is well known [33] that the computer programming of such problems is always associated with a risk to receive an erroneous result because of "natural" errors, which easily and freely "penetrate" into initial formula and programs. To avoid such errors, we propose a few tests [8], which substantially raise the level of trust to the obtained results. Note that it is the plane-wave approximation that provides useful and invaluable aid at this stage of investigations. Note also that the tests proposed below retain their validity in the case of relativistic models as well.

Let us recall that the distorted momentum distributions turn into plane-wave ones if we substitute $\psi_{K}(\boldsymbol{r}) \rightarrow \exp (i \boldsymbol{K} \boldsymbol{r})$. After that substitution the planewave overlap integral can be calculated with two different methods. The first method is to perform the limit transition of Equation (18) $\rightarrow$ Equation(A6), using the substitution $R_{K l_{1}}(r) \rightarrow j_{l_{1}}(K r)$. According to Equation (A6), we obtain:

$$
I_{x v l m}^{0}\left(\boldsymbol{q}, \boldsymbol{K}_{x}\right) \equiv\left\langle\boldsymbol{K}_{x}^{0}\left|\mathrm{e}^{i q r}\right| x v l m\right\rangle=\iiint_{-\infty}^{-i \boldsymbol{K} r} \mathrm{e}^{i q r} \varphi_{x v l m}(\boldsymbol{r}) \mathrm{d}^{3} \boldsymbol{r}
$$

$$
\begin{aligned}
& (4 \pi)^{2} \sum_{l_{1}=0}^{l_{1}=\infty} \sum_{m_{1}=-l_{1}}^{m_{1}=l_{1}=0} \sum_{l_{1}=\infty}^{l_{2}=\infty} \sum_{m_{2}=-l_{2}} i_{l_{1}} i_{l^{1}+l_{2}}(-1)^{l_{1}} \sqrt{\frac{\left(2 l_{2}+1\right)(2 l+1)}{4 \pi\left(2 l_{1}+1\right)}} \\
& \cdot\left(l_{2} 0 l 0 \mid l_{1} 0\right) \times\left(l_{2} m_{2} l m \mid l_{1} m_{1}\right) A_{l_{1} l_{2} v l}^{0}(q, K) Y_{m_{1}}^{l_{1}}\left(\boldsymbol{n}_{K}\right) Y_{m_{2}}^{l_{2}}\left(\boldsymbol{n}_{q}\right) \text {, }
\end{aligned}
$$


(A8)

where

$$
A_{l_{1} l_{2} l}^{0}(q, K)=\int_{0}^{\infty} j_{l_{1}}(K r) j_{l_{2}}(q r) R_{x v l}(r) r^{2} \mathrm{~d} r .
$$

The second method is to calculate of the overlap integral Equation (A7) in another way:

$$
\begin{aligned}
I_{x v l m}^{0} & \left(\boldsymbol{q}, \boldsymbol{K}_{x}\right) \equiv\left\langle\boldsymbol{K}_{x}^{0}\left|\mathrm{e}^{i q \boldsymbol{r}}\right| x v l m\right\rangle=\iiint_{-\infty} \mathrm{e}^{-i \boldsymbol{K} r} \mathrm{e}^{i q r} \varphi_{x v l m}(\boldsymbol{r}) \mathrm{d}^{3} \boldsymbol{r} \\
= & \iiint_{-\infty}^{i(\boldsymbol{q}-\boldsymbol{K}) \boldsymbol{r}} \varphi_{x v l m}(\boldsymbol{r}) \mathrm{d}^{3} \boldsymbol{r} \\
= & (4 \pi) i^{l} Y_{m}^{l}\left(s_{(q-K)}\right) \times \int_{0}^{\infty} j_{l}(s r) R_{v l}(r) r^{2} \mathrm{~d} r,
\end{aligned}
$$

where $\boldsymbol{s}=\boldsymbol{q}-\boldsymbol{K}, \boldsymbol{s}=|\boldsymbol{s}|$.

Note that the succession of operations Equations (A10), (A11) can be continued. All one has to do is to remember that the plane-wave momentum distribution Equation (15) is proportional to the sum

$S_{l}^{0}=\sum_{m=-l}^{m=l}\left|I_{x v l m}^{0}\left(\boldsymbol{q}, \boldsymbol{K}_{x}\right)\right|^{2}$, In this case one can as well as to recall a well-known equality [32]:

$\sum_{m=-l}^{m=l}\left|Y_{m}^{l}(\boldsymbol{n})\right|^{2}=\frac{2 l+1}{4 \pi}$. After that, the summation over $m$ in $S_{l}^{0}$ can be performed analytically:

$S_{l}^{0} \rightarrow(4 \pi)(2 l+1)\left|\int_{0}^{\infty}\left(j_{l}(s r)\right) R_{x v l}(r) r \mathrm{~d} r\right|^{2}$.

It is useful to note that for a large number $l_{1}$ $\left(l_{1} \geq 20\right)$ the strong inequality

$l_{1}\left(l_{1}+1\right) / r^{2} \gg\left|\left(2 M V_{A x}(r)\right)\right|$ takes place in the region of atomic nucleus $(r \leq R)$. It is evident that when the mentioned inequality takes place then for $l_{1}>20$ we have the approximate equality $R_{K l_{1}}(r) \approx j_{l_{1}}(K r)$, which is of high accuracy. Therefore, we can affirm that the conditions of convergence of the plane-wave approximation series and those of the series with distorted overlap integrals are identical.

Let us assume that we have established the agreement between two values of the plane-wave overlap integral calculated with the two different methods. This means that we have obtained a confirmation of reliable convergence of both series Equation (A7) and Equation (18). Note that in this way we can, at the same time, verify the convergence of expansions of the plane wave in terms of spherical functions [Equation (25)] and the distorted wave function [Equation (A2)] in the investigated kinematical region.

After calculating the overlap integrals (one after another) by two different (Equations (A8) and (A11)) methods and comparing the former and the latter results, we have established the following.

For small energies of the knocked-on protons $(1 \mathrm{MeV}$ $\leq E \leq 50 \mathrm{MeV}$, medium nuclei) it is relatively easy to achieve agreement between the plane-wave cross sections calculated by the two mentioned above methods with an accuracy of $14 \div 10$ significant figures in mantissa. Note that with the proposed comparison we achieve also a reliable accuracy check of Equations (A3) and (A8); we check also the accuracy of such special-function subroutines as $j_{l}(x), P_{m}^{l}(x)$, coefficients of ClebshGordon, as well as the accuracy of direct integration methods in the overlap integrals, etc. When the transmit- ted energy $\omega$ and, consequently, the energy $E$ of the knocked-out protons increase $(\omega, E \approx 300 \div 450 \mathrm{MeV})$, the accuracy of calculations under invariable conditions [the parameters of the calculation process such as integration step, the number of items in the sum in Equation (A7), etc. do not change] decreases: the two results coincide only with an accuracy of $6 \div 5$ significant figures in mantissa.

The good agreement between two results obtained with two different methods with high accuracy and in a wide interval of variation of the kinematic parameters gives grounds for the following assertions:

- The probability of errors and inaccuracies in our programmes and transformations of formulas in the investigated kinematic region is insignificant.

- The convergence of series in Equations (A3) and (A6) with empirically chosen boundaries of summation over quantum numbers $l_{1}, l_{2} \quad\left(l_{1}, l_{2} \leq 50\right)$ is quite satisfactory for the investigated kinematics of electrodisintegration of medium and heavy nuclei.

Let us consider one more test, which also essentially raises the reliability of the calculated numerical values of the nucleus electro-disintegration cross sections. In this case we test the process of solution of the radial Schrödinger equation [Equation (24)] and the process of tailoring of the solutions $R_{K l}^{(-)}(r)$ at the point $r \approx b$. To do this test, we should implement the special case of $V_{A x}(r)=0 \forall(E>0)$ in the subroutine solving the radial Schrödinger Equation (24). One way to do this is to put $V_{0 x}=0$ and $Z-1=0$ for the potentials $V_{W S}(r)$ and $\left.V_{C}(r)\right)$ correspondingly. In this case, if the program is correct, we must finally realize the conversions: $R_{K l}(r)=j_{l}(K r) ;\left.\Psi_{K}^{-}(r)\right|_{V_{A x}(r)=0}=\mathrm{e}^{i K r}$. Hence, the plane-wave momentum distribution calculated so must coincide with two distributions [Equations (A3) and (A7)] calculated by the traditional methods mentioned above. In our tests we obtained agreement of all distributions with an accuracy of $12 \div 6$ significant figures in mantissa in diverse regions of kinematic parameters.

Therefore, we have a right to suppose that the cross sections of nuclear electro-disintegration in the approximation of distorted waves are calculated in our computer program with sufficient accuracy. 
We have considered two tests that substantially raise the reliability of final results of our computer program. Let us suppose that we deal with large mass number $A$ and large energy $E$ of the knocked-on proton. It is instructive to mention about one more mathematical trick [8], which in this case improves the convergence of series appearing in calculations of the distorted momentum distributions. One can represent the overlap integral Equation (11) in the following form:

$$
\begin{aligned}
& I_{x v l m}\left(\boldsymbol{q}, \boldsymbol{K}_{x}\right)=\iiint_{-\infty} \psi_{K}^{(-)^{*}}(\boldsymbol{r})\left[\exp (i \boldsymbol{q} \boldsymbol{r}) \varphi_{x v l m}(\boldsymbol{r})\right] \mathrm{d}^{3} \boldsymbol{r} \\
& =\iiint_{-\infty}\left[\psi_{K}^{(-)^{*}}(\boldsymbol{r})-\exp (-i \boldsymbol{K} \boldsymbol{r})+\exp (-i \boldsymbol{K} \boldsymbol{r})\right] \\
& \cdot\left[\exp (i \boldsymbol{q} \boldsymbol{r}) \varphi_{x v l m}(\boldsymbol{r})\right] \mathrm{d}^{3} \boldsymbol{r} .
\end{aligned}
$$

Finally,

$$
\begin{aligned}
& I_{x v l m}\left(\boldsymbol{q}, \boldsymbol{K}_{x}\right)=\iiint_{-\infty}\left[\exp (i(\boldsymbol{q}-\boldsymbol{K}) \boldsymbol{r}) \varphi_{x v l m}(\boldsymbol{r})\right] \mathrm{d}^{3} \boldsymbol{r} \\
& +\iiint_{-\infty}\left[\psi_{\boldsymbol{K}}^{(-)^{*}}(\boldsymbol{r})-\exp (-i \boldsymbol{K} \boldsymbol{r})\right]\left[\exp (i \boldsymbol{q} \boldsymbol{r}) \varphi_{x v l m}(\boldsymbol{r})\right] \mathrm{d}^{3} \boldsymbol{r}
\end{aligned}
$$

method as rational as possible, for instance, like in Equations (A3)-(A11). As to the second term, we calculate it with the help of series like in Equation (A6) but with substantially improved convergence. For this purpose, one needs to perform the following substitution in Equation (A6):

$$
\begin{aligned}
& A_{l_{1} l_{2} v l}(q, K) \rightarrow A_{l_{1} l_{2} v l}^{[-]}(q, K) \\
= & \int_{0}^{\infty}\left[R_{K l_{1}}(r)-j_{l_{1}}(K r)\right] j_{l_{2}}(q r) R_{x v l}(r) r^{2} \mathrm{~d} r .
\end{aligned}
$$

This method gives a possibility to extend essentially the possibilities of numerical simulation of electro-disintegration processes of heavy nuclei for large transferred energies $\omega$. Note that all the tests described above have been taken into account, approved and implemented practically as early as in paper [7]. It is a cause for regret that all these tests do not were published so far elsewhere.

We calculate the first term of Equation (A13) with a 